\title{
TIGHT SETS IN FINITE CLASSICAL POLAR SPACES
}

\author{
ANAMARI NAKIĆ AND LEO STORME
}

\begin{abstract}
In this article we show that an $i$-tight set in the Hermitian variety $H(2 r+1, q), 81 \leq q$ odd square, is a union of pairwise disjoint $(2 r+1)$-dimensional Baer subgeometries PG $(2 r+1, \sqrt{q})$ and generators of $H(2 r+1, q)$, when $i<\left(q^{2 / 3}-1\right) / 2$. We extend the result to tight sets in the symplectic polar space $W(2 r+$ $1, q)$ and show that an $i$-tight set in $W(2 r+1, q)$ is a union of pairwise disjoint generators of $W(2 r+1, q)$, pairs of disjoint $r$ spaces $\left\{\Delta, \Delta^{\perp}\right\}$, and $(2 r+1)$-dimensional Baer subgeometries. For $W(2 r+1, q), r$ even, the possibility of pairs of disjoint $r$-spaces $\left\{\Delta, \Delta^{\perp}\right\}$ cannot occur. For the $(2 r+1)$-dimensional Baer subgeometries in the $i$-tight set of $W(2 r+1, q)$, these Baer subgeometries either are invariant under the symplectic polarity of $W(2 r+1, q)$ or they arise in pairs of disjoint Baer subgeometries which correspond to each other under the symplectic polarity of $W(2 r+1, q)$. These results are an improvement of the previous results where the upper bound on $i$ was $q^{5 / 8} / \sqrt{2}+1$. Combining the generalized version of known techniques with recent results on blocking sets and minihypers, we present an alternative proof of this result and consequently improve the upper bound on $i$ to $\left(q^{2 / 3}-1\right) / 2$. We conclude by applying our new results on tight sets to improve a known result on maximal partial spreads in the symplectic polar space $W(2 r+1, q)$.
\end{abstract}

Keywords: Tight sets; Finite classical polar spaces; Minihypers; Blocking sets; Hermitian varieties; Symplectic polar spaces

MSC 2010 codes: 05B25; 51E20

\section{Preliminaries}

Let $\mathrm{PG}(n, q)$ be the projective space of dimension $n$ over the finite field $\mathbb{F}_{q}$ of order $q=p^{h}, p$ prime. A $k$-space, $0 \leq k \leq n$, is a $k$-dimensional subspace $\mathrm{PG}(k, q)$ of $\mathrm{PG}(n, q)$. A hyperplane of $\operatorname{PG}(n, q)$ is an $(n-1)$-space. When $q$ is a square, a $k$-dimensional space $\mathrm{PG}(k, \sqrt{q}), 0<k \leq n$, defined over the subfield $\mathbb{F}_{\sqrt{q}}$ of $\mathbb{F}_{q}$, naturally embedded in $\mathrm{PG}(n, q)$, is called a $k$-dimensional Baer sub-

geometry. A hyperplane of the projective space $\mathrm{PG}(n, q)$ intersects 
an $n$-dimensional Baer subgeometry $\operatorname{PG}(n, \sqrt{q})$ in either an $(n-1)$ dimensional Baer subgeometry or in an $(n-2)$-dimensional Baer subgeometry. A $(t, k)$-Baer cone is a cone with disjoint vertex $t$-space $\mathrm{PG}(t, q)$ and base a $k$-dimensional Baer subgeometry. A $(-1, k)$-Baer cone is simply a $k$-dimensional Baer subgeometry, naturally embedded in $\mathrm{PG}(n, q)$. We shall denote $\theta_{k}=\left(q^{k+1}-1\right) /(q-1)=|\mathrm{PG}(k, q)|$ and $\theta_{k, \sqrt{q}}=\left(\sqrt{q}^{k+1}-1\right) /(\sqrt{q}-1)=|\mathrm{PG}(k, \sqrt{q})|$.

A polarity $\varphi$ of $\mathrm{PG}(n, q)$ is an involutory bijection of the set of $k$ spaces on the set of $(n-k-1)$-spaces reversing the incidence relation. A $k$-space $\Delta$ is mapped to an $(n-k-1)$-space $\Delta^{\varphi}$ called its polar space. For example, a point $P$ is mapped to its (polar) hyperplane $P^{\varphi}$. We say that points $P, R$ are conjugate if $P \in R^{\varphi}$. A point that is conjugate to itself is called self-conjugate.

Let $\mathcal{P}$ be a finite classical polar space in $\mathrm{PG}(n, q)$ of rank $r+1$. Then $\mathcal{P}$ is either the Hermitian variety $H(2 r+1, q), q$ square, the Hermitian variety $H(2 r+2, q), q$ square, the symplectic polar space $W(2 r+1, q)$, the hyperbolic quadric $Q^{+}(2 r+1, q)$, the parabolic quadric $Q(2 r+2, q)$, or the elliptic quadric $Q^{-}(2 r+3, q)$. A generator of $\mathcal{P}$ is a maximal subspace of $\mathcal{P}$, it is a subspace of dimension $r$ when $\mathcal{P}$ has rank $r+1$. If $\mathcal{P}$ is not a quadric in $\mathrm{PG}(n, q), n$ and $q$ even, then there exists a polarity $\perp$ of $\operatorname{PG}(n, q)$ such that every point $P$ of $\mathcal{P}$ satisfies $P \subseteq P^{\perp}$. Then a subspace $\Delta$ is a generator of $Q^{+}(2 r+1, q), W(2 r+1, q)$ or $H(2 r+1, q)$ if and only if $\Delta=\Delta^{\perp}$.

1.1. Blocking sets. An $(n-k)$-blocking set $B$ of $\operatorname{PG}(n, q)$ is a set of points such that any $k$-space intersects $B$ in at least one point. A 1-blocking set of $\mathrm{PG}(2, q)$ is simply called a blocking set. An $(n-k)$ blocking set $B$ is called trivial when an $(n-k)$-space is contained in $B$, otherwise $B$ is called non-trivial. Furthermore, $B$ is called minimal when no proper subset of $B$ still is an $(n-k)$-blocking set, and $B$ is called small if $|B|<\frac{3}{2}\left(q^{n-k}+1\right)$. A $t$-fold $(n-k)$-blocking set $B$ is a set of points of $\mathrm{PG}(n, q)$ intersecting every $k$-space in at least $t$ points. We say that a line is a $t$-secant of a point set $S$ if it intersects $S$ in $t$ points.

Theorem 1.1. [7] Let $B$ be an $(n-k)$-blocking set in $\mathrm{PG}(n, q)$. Then $|B| \geq \theta_{n-k}$, and equality holds if and only if $B$ is an $(n-k)$-space.

Theorem 1.2. [24] An $(n-k)$-blocking set of $\mathrm{PG}(n, q)$ of size smaller than $2 q^{n-k}$ is uniquely reducible to a minimal $(n-k)$-blocking set.

The following result was proved in [32] for the planar case, and then generalized in [33] to arbitrary dimensions. 
Theorem 1.3. [32], [33] A small minimal ( $n-k)$-blocking set of $\mathrm{PG}(n, q)$, $q=p^{h}, p$ prime, $h \geq 1$, intersects every $k$-space in $1 \bmod p$ points.

For an $(n-k)$-blocking set $B$ of $\mathrm{PG}(n, q)$, the maximal integer $e \geq 1$ such that each $k$-space intersects $B$ in $1 \bmod p^{e}$ points, is called the exponent of $B$.

Theorem 1.4. [31] Let $B$ be a small minimal $(n-k)$-blocking set of $\mathrm{PG}(n, q), q=p^{h}, p$ prime, $h \geq 1$, with exponent $e$. Then $1 \leq e$ and $e \mid h$.

From Theorem 1.1 and Theorem 1.4 follows:

Corollary 1.5. Let $B$ be a small minimal $(n-k)$-blocking set of $\mathrm{PG}(n, q), q=p^{h}, p$ prime, $h \geq 1$, with exponent $e$. Then, $e=h$ if and only if $B$ is an $(n-k)$-space.

Proof. Since the exponent of this small minimal $(n-k)$-blocking set $B$ is $e=h$, every line containing at least two points of $B$, is completely contained in $B$. This property implies that $B$ is equal to a subspace of $\mathrm{PG}(n, q)$.

Since $B$ is a small minimal $(n-k)$-blocking set of $\mathrm{PG}(n, q), B$ necessarily is an $(n-k)$-space.

Remark 1.6. The inverse statement of Theorem 1.4 is also true: for every $e \geq 1$, such that e|h, there exists a small minimal $(n-k)$-blocking set in $\mathrm{PG}(n, q), q=p^{h}, p$ prime, $h \geq 1$, with exponent $e$ (see the constructions in [25], [27], [29]). These results assure the correctness of the following notations. Let $l_{q}(n, k, e)$ and $u_{q}(n, k, e)$ denote the largest and the smallest integer such that for any minimal $(n-k)$-blocking set $B$ in $\mathrm{PG}(n, q), q=p^{h}, p$ prime, $h \geq 1$, with exponent e holds: $l_{q}(n, k, e) \leq|B| \leq u_{q}(n, k, e)$. For $p^{e} \neq 2,4,8$, the different intervals $\left[l_{q}(n, k, e), u_{q}(n, k, e)\right]$, for $1 \leq e$ and $e \mid h$, are pairwise disjoint [32], [33].

Theorem 1.7. [32], [33] Let $B$ be a small minimal $(n-k)$-blocking set of $\mathrm{PG}(n, q), q=p^{h}, 2<p$ prime. Let e be a divisor of $h$. If $|B|$ belongs to the interval $\left[l_{q}(n, k, e), u_{q}(n, k, e)\right]$, then each $k$-space intersects $B$ in $1 \bmod p^{e}$ points.

Furthermore, if $e^{\prime} \mid h$ and $e^{\prime}<e$, then $u_{q}(n, k, e)<l_{q}\left(n, k, e^{\prime}\right)$.

The following bounds are known for the planar case.

Theorem 1.8. Let $q=p^{h}, p$ prime, $h \geq 1$, and let $e \leq h / 2$ divide $h$, $p^{e} \neq 2,4,8$.

(1) $[3] q+1+p^{e}\left\lceil\frac{q / p^{e}+1}{p^{e}+1}\right\rceil \leq l_{q}(2,1, e)$. 
(2) $[28] u_{q}(2,1, e) \leq \frac{1+\left(p^{e}+1\right)(q+1)-\sqrt{\left(1+\left(p^{e}+1\right)(q+1)\right)^{2}-4\left(p^{e}+1\right)\left(q^{2}+q+1\right)}}{2}$.

(3) $[32] u_{q}(2,1, e) \leq q+9 q /\left(4 p^{e}\right)$.

(4) $[18] u_{q}(2,1, e) \leq q+a_{0} \frac{q}{p^{e}}+a_{1} \frac{q}{p^{2 e}}+\cdots+a_{h / e-2} p^{e}+1$, with $a_{i}$ the $i$-th Motzkin number.

In this article, we will use the notation $q+\epsilon$ for the size of the smallest non-trivial blocking sets in $\mathrm{PG}(2, q)$. Regarding the value $\epsilon$, the following results are known.

Theorem 1.9. Let $B$ be the smallest non-trivial blocking set in $\mathrm{PG}(2, q)$, with $|B|=q+\epsilon$.

(1) [4] If $2<q$ is a prime, then $\epsilon=(q+3) / 2$.

(2) [8] If $q$ is a square, then $\epsilon=\sqrt{q}+1$.

(3) [6] If $q$ is a non-square, $q=p^{h}, h>2, p$ prime, then $\epsilon=q^{2 / 3}+1$ for $p>3$ and $\epsilon=q^{2 / 3} / 2^{1 / 3}+1$ for $p=2,3$.

The results on the bounds on the sizes of small minimal blocking sets in $\mathrm{PG}(2, q)$ have been extended to bounds on the sizes of small minimal $(n-k)$-blocking sets in $\mathrm{PG}(n, q)$. Similarly, also a result characterizing particular collinear sets in a small minimal $(n-k)$-blocking set in $\operatorname{PG}(n, q)$ has been obtained.

Theorem 1.10. [31], [33] Let $q=p^{h}$, $p$ prime, $h \geq 1$, and let $e \leq h / 2$ divide $h, p^{e} \neq 2,4,8$.

(1) $l_{q}(n, k, e) \geq l_{q^{n-k}}(2,1, e)$ and $u_{q}(n, k, e) \leq u_{q^{n-k}}(2,1, e)$.

(2) $u_{q}(n, k, e) \leq q^{n-k}+9 q^{n-k} /\left(4 p^{e}\right)$.

(3) If a $k$-space $\Pi_{k}$ intersects a small minimal $(n-k)$-blocking set $B$ in $\mathrm{PG}(n, q)$ of exponent $e$ in precisely $p^{e}+1$ points, then the intersection $\Pi_{k} \cap B$ is a collinear point set isomorphic to a subline $\mathrm{PG}\left(1, p^{e}\right)$.

Corollary 1.11. Let $B$ be a small minimal $(n-k)$-blocking set of $\mathrm{PG}(n, q), q=p^{h}, 2<p$ prime, $h \geq 1$, with exponent $e$. Then $l_{q^{n-k}}(2,1, e) \leq|B| \leq u_{q^{n-k}}(2,1, e)$.

Moreover, $|B| \leq q^{n-k}+9 q^{n-k} /\left(4 p^{e}\right)$.

The following straightforward consequence of the two previous theorems will be often used in our arguments.

Corollary 1.12. Let $q=p^{h}, p$ prime, $h \geq 1$, and let $e \leq h / 2$ divide $h$, $p^{e} \neq 2,4,8$. Let $e$ be the exponent of a small minimal $(n-k)$-blocking set $B$ in $\mathrm{PG}(n, q)$. Then

$$
q^{k}+1+p^{e}\left\lceil\frac{q^{k} / p^{e}+1}{p^{e}+1}\right\rceil \leq|B| .
$$


We will especially be interested in non-trivial small minimal $(n-k)$ blocking sets $B$ of $\operatorname{PG}(n, q)$, when $q=p^{2 h}$ is a square and $B$ has exponent $h$, i.e. when every $k$-space intersects $B$ in $1 \bmod \sqrt{q}$ points. The nature of such point sets was studied in [34].

Theorem 1.13. [34] Let $B$ be a small minimal $(n-k)$-blocking set of $\mathrm{PG}(n, q), q=p^{2 h}, 2<p$ prime, $h \geq 1,81 \leq q$. Assume that each $k$-space intersects $B$ in $1 \bmod \sqrt{q}$ points. Then $B$ is either an $(n-k)$ space or a $(t, 2((n-k)-t-1))$-Baer cone, where $\max \{-1, n-2 k-1\} \leq$ $t<n-k-1$.

The exponent $e_{P}$ of the point $P$ of a small minimal $(n-k)$-blocking set $B$ in $\operatorname{PG}(n, q), q=p^{h}, p$ prime, is the largest integer for which each line through $P$ intersects $B$ in $1 \bmod p^{e_{P}}$ points. We note that the exponent $e_{P}$ is always larger than or equal to the exponent $e$ of the blocking set $B$. For more results on exponents of small minimal blocking sets and exponents of points, see [4], [5], [6], [31]. In our arguments we will be using the following results. In [31], the planar case has been covered. Using the technique from [33] we obtain the generalization.

Theorem 1.14. [31] Let $B$ be a small non-trivial minimal $(n-k)$ blocking set of $\mathrm{PG}(n, q)$ with exponent $e$ and $|B|=q^{n-k}+\delta$.

(1) If $P \in B$ is a point of exponent $e_{P}$, then there are at least $q^{n-k} / p^{e_{P}}-3(\delta-1) / p^{e_{P}}+2$ distinct $\left(p^{e_{P}}+1\right)$-secants of $B$ through $P$.

(2) There are at least $q^{n-k}-3 \delta+2 p^{e}+4$ points $P$ in $B$ with exponent $e_{P}=e$.

\subsection{Minihypers.}

Definition 1.15. An $\{f, m ; n, q\}$-minihyper is a pair $(F, w)$, where $F$ is a subset of the point set of $\mathrm{PG}(n, q)$ and where $w$ is a weight function $w: \operatorname{PG}(n, q) \rightarrow \mathbb{N}$, satisfying:

(1) $w(P)>0 \Leftrightarrow P \in F$,

(2) $\sum_{P \in F} w(P)=f$, and

(3) $\min \left\{\sum_{P \in H} w(P) \mid H\right.$ is a hyperplane $\}=m$.

In the case when $w$ is a mapping onto $\{0,1\}$, the minihyper $(F, w)$ can be identified with $F$ and is denoted by $F$.

The following theorem states some particular properties of minihypers.

Theorem 1.16. [22] Let $(F, w)$ be a $\left\{\sum_{i=0}^{n-1} \epsilon_{i} \theta_{i}, \sum_{i=1}^{n-1} \epsilon_{i} \theta_{i-1} ; n, q\right\}$-minihyper, where $0 \leq \epsilon_{i} \leq q-1, i=0, \ldots, n-1$, then: 
(1) If $m$ is an integer such that $1 \leq m \leq n$, then $|(F, w) \cap \Omega| \geq$ $\sum_{i=m}^{n-1} \epsilon_{i} \theta_{i-m}$ for any $(n-m)$-space $\Omega$ in $\mathrm{PG}(n, q)$ and the equality is valid for some $(n-m)$-space $\Omega$ in $\mathrm{PG}(n, q)$.

(2) $|(F, w) \cap \Delta| \geq \sum_{i=2}^{n-1} \epsilon_{i} \theta_{i-2}$ for any $(n-2)$-space $\Delta$ in $\operatorname{PG}(n, q)$ and $|(F, w) \cap G|=\sum_{i=2}^{n-1} \epsilon_{i} \theta_{i-2}$ for some $(n-2)$-space $G$ in $\operatorname{PG}(n, q)$.

Let $H_{j}, j=1,2, \ldots, q+1$, be the $q+1$ hyperplanes in $\mathrm{PG}(n, q)$ through an $(n-2)$-space $G$ intersecting $(F, w)$ in $\sum_{i=2}^{n-1} \epsilon_{i} \theta_{i-2}$ points. Then $(F, w) \cap H_{j}$ is a

$$
\left\{\delta_{j}+\sum_{i=1}^{n-1} \epsilon_{i} \theta_{i-1}, \sum_{i=2}^{n-1} \epsilon_{i} \theta_{i-2} ; n-1, q\right\} \text {-minihyper }
$$

in $H_{j}$, for $j=1,2, \ldots, q+1$, and the parameters $\delta_{j}$ are some nonnegative integers such that $\sum_{j=1}^{q+1} \delta_{j}=\epsilon_{0}$.

The following lemma, stated in [21], is a generalization of results in $[23]$.

Lemma 1.17. [21] Let $(F, w)$ be a $\left\{\sum_{i=0}^{n-1} \epsilon_{i} \theta_{i}, \sum_{i=1}^{n-1} \epsilon_{i} \theta_{i-1} ; n, q\right\}$-minihyper satisfying $n \geq 1, \epsilon_{i} \geq 0, \sum_{i=0}^{n-1} \epsilon_{i}=h \leq q$. Then every $r$-space $\Delta, 1 \leq$ $r \leq n$, not contained in $F$, intersects $(F, w)$ in a $\left\{\sum_{i=0}^{r-1} m_{i} \theta_{i}, \sum_{i=1}^{r-1} m_{i} \theta_{i-1} ; r, q\right\}$ minihyper satisfying $\sum_{i=0}^{r-1} m_{i} \leq h$.

We will also rely on the following result.

Lemma 1.18. [19] Suppose that $F$ is a $\left\{\delta \theta_{r}, \delta \theta_{r-1} ; n, q\right\}$-minihyper satisfying $0 \leq \delta \leq(q+1) / 2,0 \leq r \leq n-1$. If $H$ is a hyperplane containing more than $\delta \theta_{r-1}$ points of $F$, then $F \cap H$ is an $(n-r-1)$-blocking set of $H$.

\section{Tight SETS in Finite Classical POlaR SPACES}

2.1. Common results for $\mathcal{P}(2 r+1, q)$. In this section we present the characterization of $i$-tight sets in the Hermitian variety $H(2 r+1, q)$ and in the symplectic polar space $W(2 r+1, q)$ when $i<\left(q^{2 / 3}-1\right) / 2$. This result is the improvement of the result from [13] where the upper bound on $i$ was $q^{5 / 8} / \sqrt{2}+1$. Combining the generalized version of known techniques [26] with recent results on blocking sets and minihypers, we present an alternative proof of this result and consequently improve the upper bound on $i$ to $\left(q^{2 / 3}-1\right) / 2$.

Let $\perp$ denote the polarity corresponding to a finite classical polar space $\mathcal{P}$. 
Definition 2.1. A set of points $\mathcal{T}$ of a finite classical polar space $\mathcal{P}$ of rank $r+1$ is $i$-tight if for any point $P \in \mathcal{P}$,

$$
\left|P^{\perp} \cap \mathcal{T}\right|= \begin{cases}i \theta_{r-1}+q^{r} & , P \in \mathcal{T} \\ i \theta_{r-1} & , P \notin \mathcal{T}\end{cases}
$$

A classical example of an $i$-tight set in $\mathcal{P}$ is a union of $i$ pairwise disjoint generators of $\mathcal{P}$.

In this section the setting is the projective space $\mathrm{PG}(2 r+1, q)$ and $q=p^{2 h}$ is a square, $p$ an odd prime. By $\mathcal{P}(2 r+1, q)$ we shall commonly denote the Hermitian variety $H(2 r+1, q)$ and the symplectic polar space $W(2 r+1, q)$, and by $\mathcal{T}$ an $i$-tight set in $\mathcal{P}(2 r+1, q)$.

Lemma 2.2. [1, 13] An $i$-tight set $\mathcal{T}$ in $\mathcal{P}(2 r+1, q)$, with $i>1$, is a set of $i \theta_{r}$ points that generates the whole space $\mathrm{PG}(2 r+1, q)$ and $\mathcal{T}$ is an $\left\{i \theta_{r}, i \theta_{r-1} ; 2 r+1, q\right\}$-minihyper.

If $i=1$, then $\tau$ is a generator of $\mathcal{P}(2 r+1, q)$.

We rely on results on $m$-ovoids from [1]. An $m$-ovoid is a set $\mathcal{O}$ of points of a polar space $\mathcal{P}$ of rank $r \geq 2$, which has exactly $m$ points in common with each generator of $\mathcal{P}$. In particular, we shall use the following result.

Lemma 2.3. [1] Let $\mathcal{P}$ be a finite polar space, and let $\mathcal{O}$ and $\mathcal{T}$ be an $m$-ovoid and an $i$-tight set, respectively, of $\mathcal{P}$. Then $\mathcal{O}$ and $\mathcal{T}$ intersect in mi points.

The following lemma states the size of the intersection of an $i$-tight set with an arbitrary hyperplane of $\mathrm{PG}(2 r+1, q)$.

Lemma 2.4. If $H$ is a hyperplane of $\mathrm{PG}(2 r+1, q)$, then

$$
|H \cap \mathcal{T}|= \begin{cases}i \theta_{r-1}+q^{r} & , \text { if } H=P^{\perp} \text { for some } P \in \mathcal{T}, \\ i \theta_{r-1} & , \text { otherwise. }\end{cases}
$$

Proof. When $\mathcal{P}(2 r+1, q)$ is the symplectic polar space $W(2 r+1, q)$, every hyperplane $H$ is the polar hyperplane of some point $P$ in $W(2 r+$ $1, q)$, so the lemma immediately follows from Definition 2.1. In the case of the Hermitian variety $H(2 r+1, q)$, if $H$ is the polar hyperplane of some point $P \in H(2 r+1, q)$, then the size of the intersection with $\mathcal{T}$ is known. If $H$ is not the polar hyperplane of some point $P \in H(2 r+1, q)$, then $H \cap H(2 r+1, q)=H(2 r, q)$ is a $\theta_{r-1}$-ovoid of $H(2 r+1, q)$ and, by Lemma 2.3, we have $|H \cap \mathcal{T}|=i \theta_{r-1}$ for the hyperplanes $H, H \neq P^{\perp}$, $\forall P \in H(2 r+1, q)$. 
A hole is a point of the tight set $\mathcal{T}$. A hyperplane of $\mathrm{PG}(2 r+1, q)$ that contains $i \theta_{r-1}+q^{r}$ holes is called a rich hyperplane, otherwise it is called a poor hyperplane.

Lemma 2.5. If a $(2 r-1)$-space $\Delta$ of $\mathrm{PG}(2 r+1, q)$ is contained in $\alpha$ rich hyperplanes of $\mathcal{T}$, then $|\Delta \cap \mathcal{T}|=\alpha q^{r-1}+i \theta_{r-2}$.

Proof. Let $H_{1}, \ldots, H_{q+1}$ be the hyperplanes of $\mathrm{PG}(2 r+1, q)$ through $\Delta$. Then

$$
\sum_{j=1}^{q+1}\left|H_{j} \cap \mathcal{T}\right|=\alpha\left(q^{r}+i \theta_{r-1}\right)+(q+1-\alpha) i \theta_{r-1} .
$$

It follows that

$$
|\mathcal{T}|+q|\Delta \cap \mathcal{T}|=\alpha\left(q^{r}+i \theta_{r-1}\right)+(q+1-\alpha) i \theta_{r-1},
$$

and, consequently,

$$
|\Delta \cap \mathcal{T}|=\alpha q^{r-1}+i \theta_{r-2}
$$

Suppose now that $i \leq(q+1) / 2$. If a hyperplane $H$ of $\mathrm{PG}(2 r+1, q)$ is a rich hyperplane, then by Lemma $1.18, H \cap \mathcal{T}$ is an $r$-blocking set

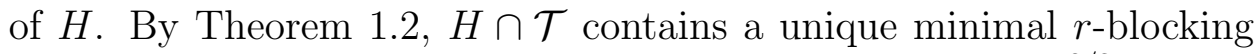
set $B$. In the following two lemmas, we show that if $i<\left(q^{2 / 3}-1\right) / 2$, this minimal $r$-blocking set $B$ is a $(2 r)$-dimensional Baer subgeometry, naturally embedded in $H$, when $B$ is non-trivial.

Lemma 2.6. Let $B$ be a non-trivial minimal $r$-blocking set in $\mathrm{PG}(2 r, q)$, $q=p^{2 h}, 2<p, h \geq 1$, with exponent e. If $|B|<q^{r}+\frac{1}{2} q^{r-1 / 3}+1$, then $e=h$.

Proof. The point set $B$ is a small minimal $r$-blocking set with exponent $e$, so by Theorem $1.10, e \mid(2 h)$. Furthermore, since $B$ is a non-trivial blocking set, by Corollary 1.5, e $<2 h$. The largest possible value of $e$ therefore is $2 h / 2=h$. Let $e^{\prime}$ denote the second largest possible value of $e$. Obviously, $e^{\prime} \leq 2 h / 3<h$. By Theorem 1.7 and Remark 1.6, from $e^{\prime}<h$ follows

$$
l_{q}\left(2 r, r, e^{\prime}\right)>u_{q}(2 r, r, h) \geq l_{q}(2 r, r, h) .
$$

Hence, showing that $|B|<l_{q}\left(2 r, r, e^{\prime}\right)$ would imply that $e=h$. Again, by Theorem 1.7 and Remark 1.6, it is sufficient to discuss the case $3 \mid(2 h)$ with $e^{\prime}=2 h / 3$ and by Corollary 1.12 ,

$$
l_{q}(2 r, r, 2 h / 3) \geq q^{r}+1+p^{2 h / 3}\left\lceil\frac{q^{r} / p^{2 h / 3}+1}{p^{2 h / 3}+1}\right\rceil .
$$


But

$$
q^{r}+1+p^{2 h / 3}\left\lceil\frac{q^{r} / p^{2 h / 3}+1}{p^{2 h / 3}+1}\right\rceil \geq q^{r}+\frac{1}{2} q^{r-1 / 3}+1>|B| .
$$

Therefore, $e>2 h / 3$ and consequently, $e=h$.

If $B$ is a non-trivial small minimal $r$-blocking set of $\mathrm{PG}(2 r, q)$ with exponent $h$, then every $r$-space intersects $B$ in $1 \bmod \sqrt{q}$ points. We are now interested in the nature of such $r$-blocking sets when all of their points are holes of a given tight set $\mathcal{T}$ of $\mathcal{P}(2 r+1, q)$.

Lemma 2.7. Let $\mathcal{T}$ be an $i$-tight set in $\mathcal{P}(2 r+1, q), i \leq(q+1) / 2$, $q=p^{2 h}, 2<p, 81 \leq q$. Let $B \subset \mathcal{T}$ be a non-trivial small minimal $r$-blocking set of a rich hyperplane $H$, with exponent $h$. Then $B$ is a (2r)-dimensional Baer subgeometry, naturally embedded in $H$.

Proof. By Theorem 1.13, $B$ is a $(t, 2 r-2 t-2)$-Baer cone $\mathcal{C},-1 \leq t<$ $r-1$, with $t$-dimensional vertex $\pi_{t}$ and base a $(2 r-2 t-2)$-dimensional Baer subgeometry, naturally embedded in a $(2 r-2 t-2)$-space $\pi_{2 r-2 t-2}$ skew to $\pi_{t}$. Here, in this proof, we fix the space $\pi_{2 r-2 t-2}$ containing the base of $B$. We shall prove that necessarily $t=-1$, and therefore $B$ is a $(2 r)$-dimensional Baer subgeometry, naturally embedded in $H$.

The hyperplane $H$ is a rich hyperplane of $\mathcal{T}$, therefore

$$
|H \cap \mathcal{T}|=q^{r}+i \theta_{r-1} .
$$

Denote by $E$ the number of holes in $H \backslash B$ :

$$
E=|(H \cap \mathcal{T}) \backslash B|=q^{r}+i \theta_{r-1}-|B|
$$

Our strategy in this proof is the following: we shall obtain a lower and an upper bound on $E$ and then show that necessarily $t=-1$.

An upper bound on E. By Corollary 1.12,

$$
|B| \geq q^{r}+1+\sqrt{q}\left\lceil\frac{q^{r} / \sqrt{q}+1}{\sqrt{q}+1}\right\rceil \text {. }
$$

Introducing (2) in (1), we obtain

$$
E \leq i \theta_{r-1}
$$

A lower bound on $E$. Consider a plane $\Delta=\langle P, L\rangle$ generated by a point $P$ in the vertex $\pi_{t}$ of $B$ and a line $L$ intersecting the base of $B$ in a Baer subline $\Omega_{1}$. The intersection of $B$ and $\Delta$ is a unique Baer cone $\mathcal{E}$ with $P$ as a vertex and with the Baer subline $\Omega_{1}$ as a base. This setting is visualised in Figure 1. 


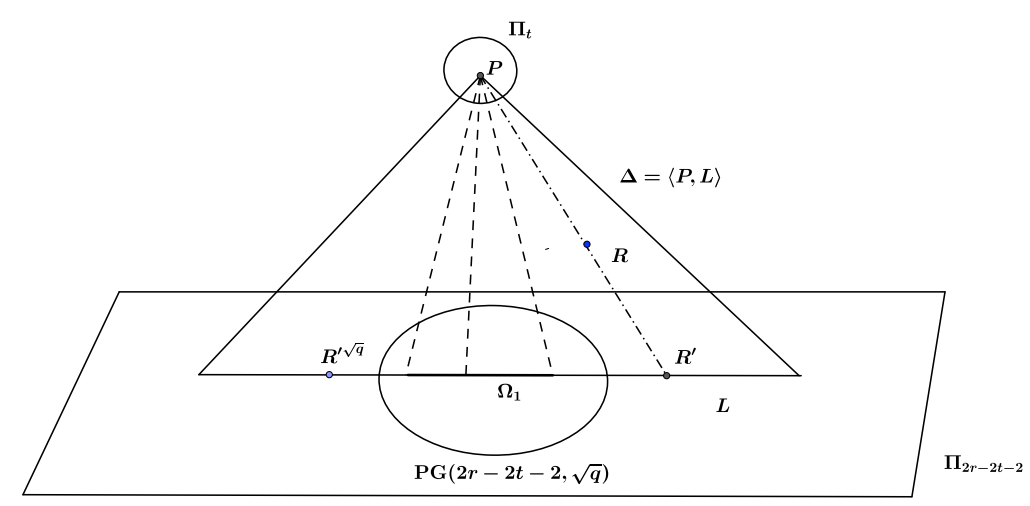

Figure 1. Baer cone $B$ and plane $\Delta$

We are interested in deriving a lower bound on the number $E_{\Delta}$ of holes in $\Delta$ outside of $\Delta \cap B$;

$$
E_{\Delta}=|(\Delta \cap \mathcal{T}) \backslash B|=|(\Delta \cap \mathcal{T}) \backslash \mathcal{E}|
$$

First we notice that

$$
|\Delta \cap \mathcal{T}| \geq|\mathcal{E}|=(\sqrt{q}+1)(q+1)-\sqrt{q} .
$$

The tight set $\mathcal{T}$ is an $\left\{i \theta_{r}, i \theta_{r-1} ; 2 r+1, q\right\}$-minihyper (Lemma 2.2). If $\Delta \nsubseteq \mathcal{T}$, then $\Delta \cap \mathcal{T}$ is an $\left\{m_{1}(q+1)+m_{0}, m_{1} ; 2, q\right\}$-minihyper, with $m_{1}+m_{0} \leq i, m_{0}, m_{1} \geq 0$ (Lemma 1.17). We note that $m_{1} \geq \sqrt{q}+1$. Namely, introducing $m_{1} \leq \sqrt{q}$ in (4), we obtain that $m_{1}+m_{0} \geq q+1>$ $i$; a contradiction.

From the definition of a minihyper, it follows that $\Delta \cap \mathcal{T}$ is an $m_{1}$-fold blocking set in $\Delta$. Hence, the remaining $(q+1)-(\sqrt{q}+1)=q-\sqrt{q}$ lines of $\Delta \backslash \mathcal{E}$ through $P$ each share at least $\sqrt{q}+1$ points with $\mathcal{T}$. So, in total, $\Delta \backslash \mathcal{E}$ shares at least $(q-\sqrt{q})(\sqrt{q}-1)$ extra holes with $\mathcal{T}$ not lying on $L$ :

$$
E_{\Delta} \geq|(\Delta \cap \mathcal{T}) \backslash \mathcal{E}|-|L \cap \mathcal{T}| \geq(q-\sqrt{q})(\sqrt{q}-1) .
$$

A hole $R$ in $H \backslash B$ belongs to at most one such plane $\Delta$. Namely, project such a hole $R$ from the vertex $\pi_{t}$ onto a $(2 r-t)$-space $\pi_{2 r-t}$ through the space $\pi_{2 r-2 t-2}$, skew to $\pi_{t}$. Then, the projection of $R$ is a point $R^{\prime}$ not belonging to the base of $B$. If $R^{\prime} \in \pi_{2 r-2 t-2}$, then there is a unique line $L$ of $\pi_{2 r-2 t-2}$ that contains $R^{\prime}$ and that intersects the base of $B$ in a Baer subline $\Omega_{1}$. This line $L$ is the line pairing $R^{\prime}$ to its 
conjugate point $R^{\prime \sqrt{q}}$ with respect to the $(2 r-2 t-2)$-dimensional Baer subgeometry in $\pi_{2 r-2 t-2}$ which is the base of $B$. Thus, $R$ is contained in at most one plane $\Delta=\langle P, L\rangle$, with $P$ belonging to the vertex $\pi_{t}$ of $B$ and with $L$ a line of $\pi_{2 r-2 t-2}$ which intersects the base of $B$ in a Baer subline $\Omega_{1}$.

The number of lines $L$ equals the number of Baer sublines in the $(2 r-2 t-2)$-dimensional Baer subgeometry forming the base of $B$, that is, equals $\theta_{r-t-2} \theta_{2 r-2 t-2, \sqrt{q}}$. Each line $L$ gives $\theta_{t}$ such planes $\langle P, L\rangle$, with $P \in \pi_{t}$, and each such plane $\langle P, L\rangle$ gives at least $(q-\sqrt{q})(\sqrt{q}-1)$ extra holes not in $B$ and not belonging to $L$. Therefore,

$$
E \geq \theta_{r-t-2} \theta_{2 r-2 t-2, \sqrt{q}} \theta_{t}(q-\sqrt{q})(\sqrt{q}-1) .
$$

Finally, taking into consideration the upper bound (3) and the lower bound (5) on $E$, as well as the upper bound $i \leq(q+1) / 2$ on $i$, we obtain that

$$
\theta_{r-t-2} \theta_{2 r-2 t-2, \sqrt{q}} \theta_{t}(q-\sqrt{q})(\sqrt{q}-1) \leq \theta_{r-1}(q+1) / 2,
$$

which implies that $r-t=1$. But the equality $r-t=1$ contradicts the inequality $t<r-1$ from Theorem 1.13. Therefore, $t=-1$ and $B$ is a (2r)-dimensional Baer subgeometry, naturally embedded in $H$.

The next corollary now easily follows from the two previous lemmas.

Corollary 2.8. Let $\mathcal{T}$ be an $i$-tight set in $\mathcal{P}(2 r+1, q), 81 \leq q$ odd square, containing no $r$-spaces, $i<\left(q^{2 / 3}-1\right) / 2$. Then every rich hyperplane $H$ shares a unique $(2 r)$-dimensional Baer subgeometry $B_{H}$ with $\mathcal{T}$, naturally embedded in $H$.

Proof. First note that $i<\left(q^{2 / 3}-1\right) / 2 \leq(q+1) / 2$. If a hyperplane $H$ of $\mathrm{PG}(2 r+1, q)$ is a rich hyperplane, then by Lemma $1.18, H \cap \mathcal{T}$ is an $r$-blocking set of $H$. By Theorem 1.2, $H \cap \mathcal{T}$ contains a unique minimal $r$-blocking set $B_{H}$ with exponent $e$. Furthermore, $\left|B_{H}\right| \leq|H \cap \mathcal{T}|=$ $q^{r}+i \theta_{r-1}<q^{r}+\frac{1}{2} q^{r-1 / 3}+1$. Therefore, by Lemma 2.6, the exponent of $B_{H}$ equals $e=h$. By Lemma 2.7, $B_{H}$ is a $(2 r)$-dimensional Baer subgeometry, naturally embedded in $H$.

Please note that in the previous corollary we only state that $B_{H} \subseteq$ $H \cap \mathcal{T}$. We will prove more specialized results on these $(2 r)$-dimensional Baer subgeometries $B_{H}$ of holes contained in the rich hyperplanes $H$ in Lemmas 2.11 and 2.12.

Now we note the dual setting of the previous corollary.

Lemma 2.9. Let $\mathcal{T}$ be an $i$-tight set in $\mathcal{P}(2 r+1, q), 81 \leq q$ odd square, containing no $r$-spaces, $i<\left(q^{2 / 3}-1\right) / 2$. Then the rich hyperplanes 


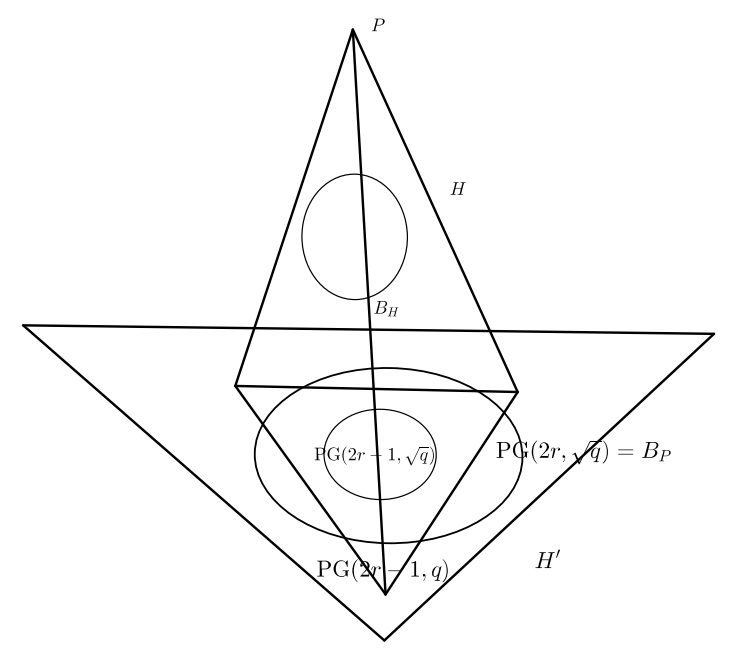

Figure 2. Dual setting of Corollary 2.8

through a hole $P$ contain a (dual) (2r)-dimensional Baer subgeometry $B_{P}^{*}$, naturally embedded in the quotient geometry $\mathrm{PG}(2 r+1, q)_{P}$ of $P$.

Proof. Let $H=P^{\perp}$ and let $B_{H}$ be the $(2 r)$-dimensional Baer subgeometry of holes, naturally embedded in $H$ (Corollary 2.8). Then $B_{H}^{\perp}=B_{P}^{*}$ is a dual $(2 r)$-dimensional Baer subgeometry of rich hyperplanes, naturally embedded in the quotient geometry $\mathrm{PG}(2 r+1, q)_{P}$ of $P$.

Remark 2.10. Figure 2 illustrates this dual setting. The main goal of the explanation which follows is to show that it is possible to describe the dual Baer subgeometry $B_{P}^{*}$ of rich hyperplanes through $P$ much easier in an equivalent way by a $(2 r)$-dimensional Baer subgeometry $B_{P}$ of points in a fixed hyperplane $H^{\prime}$ not through $P$. Namely, this dual Baer subgeometry $B_{P}^{*}$ of rich hyperplanes through $P$ is also obtained in the following way: consider a fixed hyperplane $H^{\prime}$ of $\mathrm{PG}(2 r+1, q)$ not containing $P$. In this hyperplane $H^{\prime}$, there is a particular $(2 r)$ dimensional Baer subgeometry $B_{P}$ satisfying the following properties.

(1) Consider a Baer hyperplane, i.e. a hyperplane of $H^{\prime}$ sharing a $(2 r-1)$-dimensional Baer subgeometry with $B_{P}$.

(2) Extend this Baer hyperplane to a $(2 r-1)$-space defined over $\mathbb{F}_{q}$.

(3) Then, by adding the point $P$, a hyperplane $H$ of the dual (2r)dimensional Baer subgeometry $B_{P}^{*}$ of rich hyperplanes through $P$ is obtained. 
Figure 2 shows this fixed hyperplane $H^{\prime}$ containing the $(2 r)$-dimensional Baer subgeometry $B_{P}$, the rich hyperplane $H$ and the $(2 r)$-dimensional Baer subgeometry $B_{H}$ of holes in this rich hyperplane $H$.

$W e$ shall denote by $\mathcal{C}$ the cone with vertex $P$ and base the $(2 r)$ dimensional Baer subgeometry $B_{P}$. With the hyperplanes of $\mathcal{C}$, we denote the rich hyperplanes through $P$ described above in (1), (2) and (3). The lines of $\mathcal{C}$ are the lines through $P$ and a point of $B_{P}$.

Note that all these hyperplanes $H$ of $\mathcal{C}$ are rich, so by Corollary 2.8, they contain a $(2 r)$-dimensional Baer subgeometry $B_{H}$, naturally embedded in $H$, completely consisting of holes.

The following properties will be proven in the next two lemmas regarding these $(2 r)$-dimensional Baer subgeometries $B_{H}$ of holes, defined by the hyperplanes $H$ of $\mathcal{C}$ :

- for all hyperplanes $H$ of $\mathcal{C}, P \in B_{H}$ (Lemma 2.11);

- for all hyperplanes $H$ of $\mathcal{C}, B_{H} \subseteq \mathcal{C}$ (Lemma 2.12).

We also recall that a hyperplane $H$ of $\mathcal{C}$ contains $\theta_{2 r-1, \sqrt{q}}$ lines of $\mathcal{C}$ that define a $(2 r-1)$-dimensional Baer subgeometry of the $(2 r)$-dimensional Baer subgeometry $B_{P}$. Two such rich hyperplanes of $\mathcal{C}$ share $\theta_{2 r-2, \sqrt{q}}$ lines of $\mathcal{C}$. The Baer cone $\mathcal{C}$ contains in total $\theta_{2 r, \sqrt{q}}$ lines of $\mathcal{C}$.

We note already that our goal is to prove that the hole $P$ belongs to a $(2 r+1)$-dimensional Baer subgeometry of holes completely lying within this Baer cone $\mathcal{C}$. The desired goal of proving that $P$ belongs to a $(2 r+1)$-dimensional Baer subgeometry of holes lying on the cone $\mathcal{C}$ is achieved in Lemma 2.13, following the two preparatory lemmas 2.11 and 2.12 .

Lemma 2.11. Let $\mathcal{T}$ be an $i$-tight set of $\mathcal{P}(2 r+1, q), i<\left(q^{2 / 3}-\right.$ 1)/2. Consider a hole $P \in \mathcal{T}$ and the Baer cone $\mathcal{C}$ defined by the rich hyperplanes through $P$. Let $H$ be a hyperplane of $\mathcal{C}$ and let $B_{H}$ be the $(2 r)$-dimensional Baer subgeometry that $H$ shares with $\mathcal{T}$. Then $P$ is a point of $B_{H}$.

Proof. Figure 3 shows the setting of Lemma 2.11.

Let us assume the opposite: $P \notin B_{H}$. Then $P$ has a conjugate point $P^{\sqrt{q}} \neq P$ with respect to $B_{H}$.

In our proof we shall use particular $\theta_{2 r-1, \sqrt{q}}$ distinct $(2 r-1)$-spaces $\Delta$ in $H$ through $P$. We shall describe them in the following lines. The hyperplane $H$ intersects the base of $\mathcal{C}$ in a $(2 r-1)$-dimensional Baer subgeometry $\Omega$. There are $\theta_{2 r-1, \sqrt{q}}(2 r-2)$-dimensional Baer subgeometries in $\Omega$. We extend a $(2 r-2)$-dimensional Baer subgeometry in $\Omega$ over $\mathbb{F}_{q}$ to obtain a $(2 r-2)$-space $G$ in $H$. Then $\Delta=\langle P, G\rangle$ 


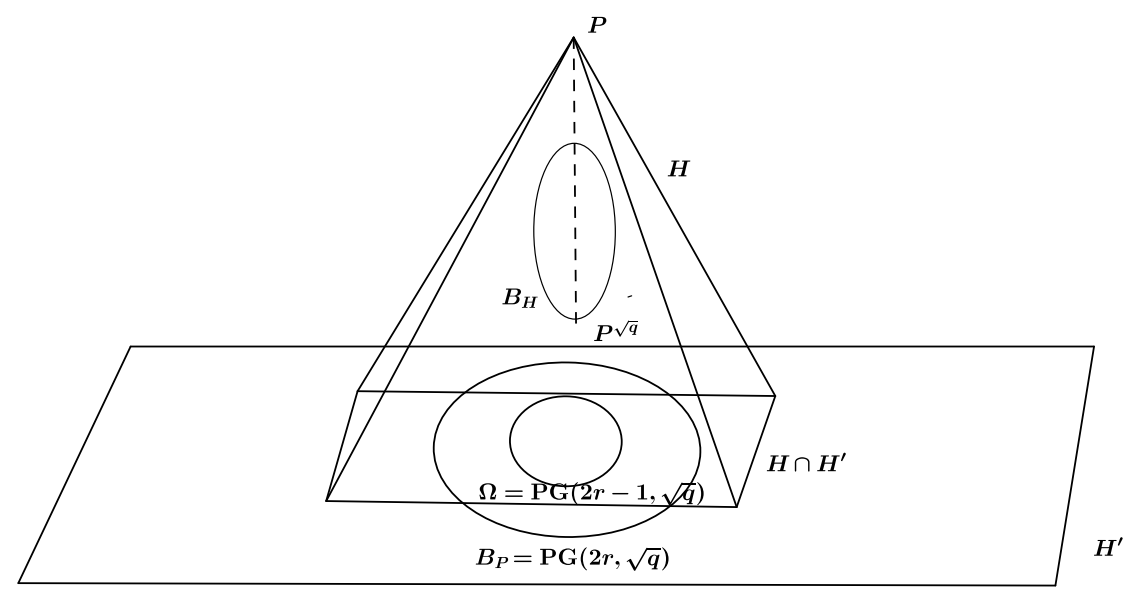

FiguRE 3. Setting of Lemma 2.11

is a $(2 r-1)$-space in $H$. Furthermore, from the properties of Baer subgeometries, it follows that $\Delta \cap B_{H}$ is either a $(2 r-2)$-dimensional or a $(2 r-1)$-dimensional Baer subgeometry [30].

We shall now count the number of $(2 r-1)$-spaces $\Delta$ of $H$ that intersect $B_{H}$ in a $(2 r-1)$-dimensional Baer subgeometry. We note the following. If $\Delta \cap B_{H}$ is a $(2 r-1)$-dimensional Baer subgeometry, then $\Delta$ necessarily contains the line $P P^{\sqrt{q}}$. The line $P P^{\sqrt{q}}$ intersects $B_{H}$ in a unique Baer subline that is contained in $\theta_{2 r-2, \sqrt{q}}(2 r-1)$-dimensional Baer subgeometries in $B_{H}$.

Therefore, at most $\theta_{2 r-2, \sqrt{q}}$ distinct $(2 r-1)$-spaces $\Delta$ share a $(2 r-1)$ dimensional Baer subgeometry with $B_{H}$. The remaining $(2 r-1)$-spaces $\Delta$ share a $(2 r-2)$-dimensional Baer subgeometry with $B_{H}$ : denote them by $\Delta_{j}=\left\langle P, G_{j}\right\rangle, G_{j}=H^{\prime} \cap \Delta_{j}, j=1, \ldots, \sqrt{q}^{2 r-1}, \ldots$ Let $d_{j}$ denote the number of holes in $\Delta_{j} \backslash B_{H}$. The space $\Delta_{j}$ is contained in $\sqrt{q}+1$ hyperplanes of $\mathcal{C}$ since it intersects $H^{\prime}$ in a $(2 r-2)$-space $G_{j}$ which intersects $B_{P}$ in a $(2 r-2)$-dimensional Baer subgeometry. Note that this $(2 r-2)$-space $G_{j}$ corresponds to the space $G$ initially defined to construct the $(2 r-1)$-space $\Delta$. Here, $G_{j}$ lies in $\sqrt{q}+1$ hyperplanes of $H^{\prime}$ intersecting $B_{P}$ in $(2 r-1)$-dimensional Baer subgeometries, which, together with $P$, define the $\sqrt{q}+1$ hyperplanes of $\mathcal{C}$ through $\Delta_{j}$. Every hyperplane of $\mathcal{C}$ through $\Delta_{j}$ is rich, so, by Lemma 2.5 with $\alpha \geq \sqrt{q}+1$, 
$\left|\Delta_{j} \cap \mathcal{T}\right| \geq(\sqrt{q}+1) q^{r-1}+i \theta_{r-2}$. Therefore,

$$
d_{j} \geq(\sqrt{q}+1) q^{r-1}+i \theta_{r-2}-\theta_{2 r-2, \sqrt{q}}
$$

and

(7)

$$
\sum_{j} d_{j}-\sqrt{q}^{2 r-1} \geq \sqrt{q}^{2 r-1}\left[(\sqrt{q}+1) q^{r-1}+i \theta_{r-2}-\theta_{2 r-2, \sqrt{q}}\right]-\sqrt{q}^{2 r-1} .
$$

The term $\sqrt{q}^{2 r-1}$ on the left hand side is subtracted since we do not count the point $P$ which is contained in every $(2 r-1)$-space $\Delta_{j}$.

Consider a hole $R \in H \backslash B_{H}, R \neq P$. Then, such a $(2 r-1)$-space $\Delta_{j}$ contains such a hole $R$ if and only if it contains the projection $R^{\prime}$ of $R$ from $P$ onto $H^{\prime}$. If $R^{\prime} \in \Omega$, then $R^{\prime}$ belongs to $\theta_{2 r-2, \sqrt{q}}$ such $(2 r-1)$-spaces in $H \cap H^{\prime}$. If $R^{\prime} \notin \Omega$, then $R^{\prime} \in H^{\prime} \cap \Delta_{j}=G_{j}$, and then such a $(2 r-1)$-space $\Delta_{j}$ also contains the conjugate point $R^{\prime \sqrt{q}}$ with respect to $\Omega$. The line $R^{\prime} R^{\prime \sqrt{q}}$ intersects $\Omega$ in a unique Baer subline that is contained in $\theta_{2 r-3, \sqrt{q}}$ such $(2 r-1)$-spaces in $H \cap H^{\prime}$. Therefore, $R^{\prime} \notin \Omega$, and consequently, also $R$ is contained in $\theta_{2 r-3, \sqrt{q}}$ such $(2 r-1)$-spaces $\Delta_{j}$. We see that $R$ contributes the most if $R^{\prime} \in \Omega$, SO

$$
\sum_{j} d_{j}-\sqrt{q}^{2 r-1} \leq\left|(H \cap \mathcal{T}) \backslash B_{H}\right| \theta_{2 r-2, \sqrt{q}} .
$$

Now, the inequalities (7) and (8) yield

$$
\sqrt{q}^{2 r-1}\left[\sqrt{q}^{2 r-1}-1+(i-\sqrt{q}-1) \theta_{r-2}\right] \leq(i-\sqrt{q}-1) \theta_{r-1} \theta_{2 r-2, \sqrt{q}},
$$

implying that $i \geq q / 2$; a contradiction.

The proof of the following lemma is a generalization of the technique used in the proof of Lemma 2.2 in [26], which discusses the case $r=1$.

Lemma 2.12. Let $\mathcal{T}$ be an $i$-tight set in $\mathcal{P}(2 r+1, q), 81 \leq q$ odd square, containing no $r$-spaces, $i<\left(q^{2 / 3}-1\right) / 2$. Consider a hole $P$ and the Baer cone $\mathcal{C}$ with vertex $P$ defined by the rich hyperplanes $H$ through $P$. Then, for a rich hyperplane $H$ of the Baer cone $\mathcal{C}$, the unique $(2 r)$-dimensional Baer subgeometry $B_{H}$ that $H$ shares with $\mathcal{T}$ lies completely within the cone $\mathcal{C}$.

Proof. Denote by $H_{j}, j=1, \ldots, \theta_{2 r, \sqrt{q}}$, the hyperplanes of $\mathcal{C}$. Every hyperplane $H_{j}$ shares a unique $(2 r)$-dimensional Baer subgeometry $B_{j}$ with $\mathcal{T}$ and $P$ is a point of $B_{j}$ (Corollary 2.8 and Lemma 2.11). Suppose that there exists a hyperplane $H_{l}$ of the Baer cone $\mathcal{C}$ such that $B_{l} \not \subset \mathcal{C}$. Let $E$ be the number of such hyperplanes $H_{l}$ for which $B_{l} \not \subset \mathcal{C}$.

If $a_{j}$ is the number of holes in $H_{j} \backslash \mathcal{C}$, then 


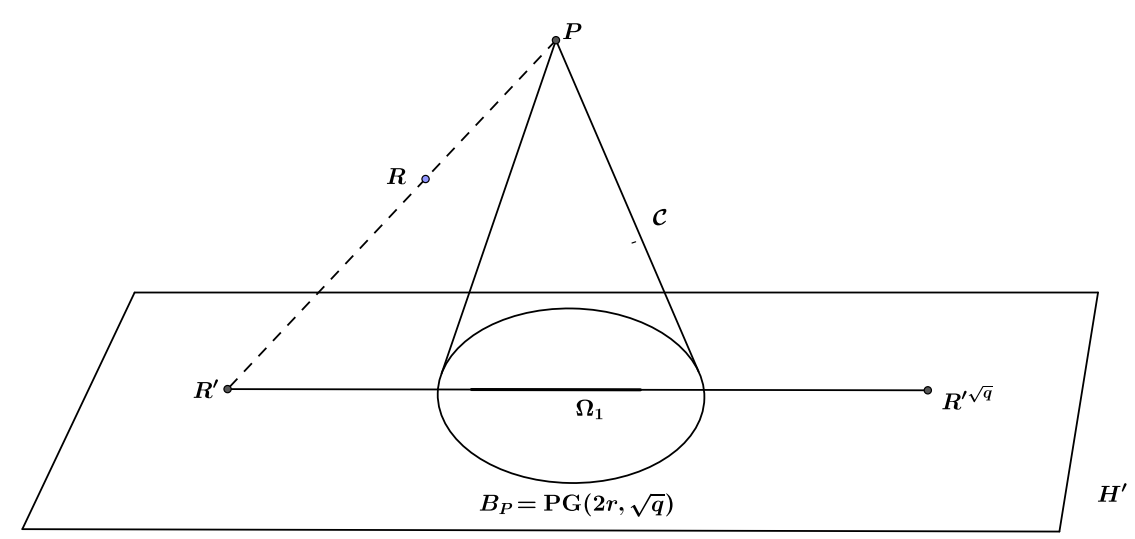

FiguRE 4. The setting of Lemma 2.12

$$
\sum_{j} a_{j}=q \theta_{2 r-2, \sqrt{q}} \theta_{2 r-1, \sqrt{q}}(i-\sqrt{q}-1) /(\sqrt{q}+1) .
$$

The equality (9) is obtained from the following equality:

$$
|\mathcal{T}|=1+\frac{1}{\theta_{2 r-2, \sqrt{q}}} \sum_{j} a_{j}+\frac{1}{\theta_{2 r-1, \sqrt{q}}} \sum_{j}\left(\left|H_{j} \cap \mathcal{T}\right|-a_{j}-1\right) .
$$

To obtain the right hand side of the previous expression, we take into account that a hole $R \neq P$ belongs to multiple hyperplanes of $\mathcal{C}$. The setting is visualised in Figure 4. A hyperplane of $\mathcal{C}$ contains $R$ if and only if it contains its projection $R^{\prime}$ from $P$ onto the hyperplane $H^{\prime}$ that contains the base $B_{P}$ of $\mathcal{C}$. If $R \in \mathcal{C}$, then the projection $R^{\prime}$ belongs to the base $B_{P}$ of $\mathcal{C}$. There are $\theta_{2 r-1, \sqrt{q}}$ distinct $(2 r-1)$-dimensional Baer subgeometries through $R^{\prime}$ in the base $B_{P}$ of $\mathcal{C}$, and each such $(2 r-1)$-dimensional Baer subgeometry belongs to the intersection of the base $B_{P}$ of $\mathcal{C}$ with a unique hyperplane of $\mathcal{C}$. Therefore, $R$ belongs to $\theta_{2 r-1, \sqrt{q}}$ hyperplanes of the cone $\mathcal{C}$. If $R \notin \mathcal{C}$, then its projection $R^{\prime}$ lies outside of the base $B_{P}$ of $\mathcal{C}$ in the hyperplane $H^{\prime}$. The line $R^{\prime} R^{\prime \sqrt{q}}$ intersects the base $B_{P}$ of $\mathcal{C}$ in a unique Baer subline $\Omega_{1}$ that is contained in $\theta_{2 r-2, \sqrt{q}}$ distinct $(2 r-1)$-dimensional Baer subgeometries of the base $B_{P}$ of $\mathcal{C}$. Therefore, $R$ belongs to $\theta_{2 r-2, \sqrt{q}}$ hyperplanes of the cone $\mathcal{C}$. Applying these observations we obtain (10), whereby we 
count the point $P$ separately since $P$ belongs to all the hyperplanes of $\mathcal{C}$.

Now we show that if $a_{j}<q^{r}-\sqrt{q}$, then $B_{j} \subseteq \mathcal{C}$. If $a_{j}<q^{r}-\sqrt{q}$, then

$$
\left|B_{j} \cap \mathcal{C}\right|=\left|B_{j}\right|-a_{j}>\sqrt{q}\left(\theta_{2 r-2, \sqrt{q}}+1\right)+1 .
$$

The hyperplane $H_{j}$ contains $\theta_{2 r-1, \sqrt{q}}$ lines of $\mathcal{C}$ through $P$ with the property: the intersection of these lines and $H^{\prime}$ is a $(2 r-1)$-dimensional Baer subgeometry $\Omega_{j}$. Each of those lines intersects the Baer subgeometry $B_{j}$ of holes in $H_{j}$ in 1 or $\sqrt{q}+1$ points since $P \in B_{H}$. By (11), more than $\theta_{2 r-2, \sqrt{q}}+1$ of those lines of $\mathcal{C}$ in $H_{j}$ are $(\sqrt{q}+1)$-secants of $B_{j}$. On the other hand, $P$ belongs to $\theta_{2 r-1, \sqrt{q}}$ lines of $H_{j}$ that are $(\sqrt{q}+1)$-secants of $B_{j}$ and which define a $(2 r-1)$-dimensional Baer subgeometry $\Omega_{j}^{\prime}$ in the quotient geometry of $P$. These two $(2 r-1)$-dimensional Baer subgeometries $\Omega_{j}$ and $\Omega_{j}^{\prime}$ intersect in more than $\theta_{2 r-2, \sqrt{q}}+1$ points, therefore they must be equal [16]. Hence, every line of $\mathcal{C}$ in $H_{j}$ shares a Baer subline with $B_{j}$ and $B_{j} \subseteq \mathcal{C}$. Thus, if $B_{l} \nsubseteq \mathcal{C}$, then $a_{l} \geq q^{r}-\sqrt{q}$, and, consequently,

$$
\left|B_{l} \cap \mathcal{C}\right| \leq i \theta_{r-1}+\sqrt{q} .
$$

If we denote by $L$ the number of lines of $\mathcal{C}$ in $H_{l}$ that contain less than $\sqrt{q}+1$ holes, then

$$
L \geq \theta_{2 r-1, \sqrt{q}}-\left(i \theta_{r-1}+\sqrt{q}\right) / \sqrt{q} .
$$

Namely, at most $i \theta_{r-1}+\sqrt{q}$ holes of $H_{l}$ belong also to $\mathcal{C}$, so, since $P \in B_{l}$, at most $\left(i \theta_{r-1}+\sqrt{q}\right) / \sqrt{q}$ lines of $\mathcal{C}$ in $H_{l}$ contain at least $\sqrt{q}+1$ holes.

If a line of $\mathcal{C}$, lying in $H_{l}$, contains less than $\sqrt{q}+1$ holes, then for every hyperplane $H_{l^{\prime}}$ of the Baer cone $\mathcal{C}$ through this line, necessarily $B_{l^{\prime}} \nsubseteq \mathcal{C}$. There are exactly $\theta_{2 r-1, \sqrt{q}}-1$ such hyperplanes $H_{l^{\prime}}$, other than $H_{l}$, through a given line of $\mathcal{C}$, lying in $H_{l}$. Every such hyperplane $H_{l^{\prime}}$ shares $\theta_{2 r-2, \sqrt{q}}$ lines of $\mathcal{C}$ with $H_{l}$. So we obtain via the preceding double counting argument that

$$
1+L \cdot\left(\theta_{2 r-1, \sqrt{q}}-1\right) / \theta_{2 r-2, \sqrt{q}} \leq E,
$$

where the 1 corresponds to the fixed hyperplane $H_{l}$ for which $B_{l} \not \subset \mathcal{C}$.

Double counting of the set

$$
\left\{\left(R, H_{l^{\prime}}\right) \mid R \in \mathcal{T} \backslash \mathcal{C}, R \in H_{l^{\prime}}, B_{l^{\prime}} \nsubseteq \mathcal{C}\right\}
$$

yields

$$
E \cdot\left(q^{r}-\sqrt{q}\right) \leq \sum_{j} a_{j}
$$




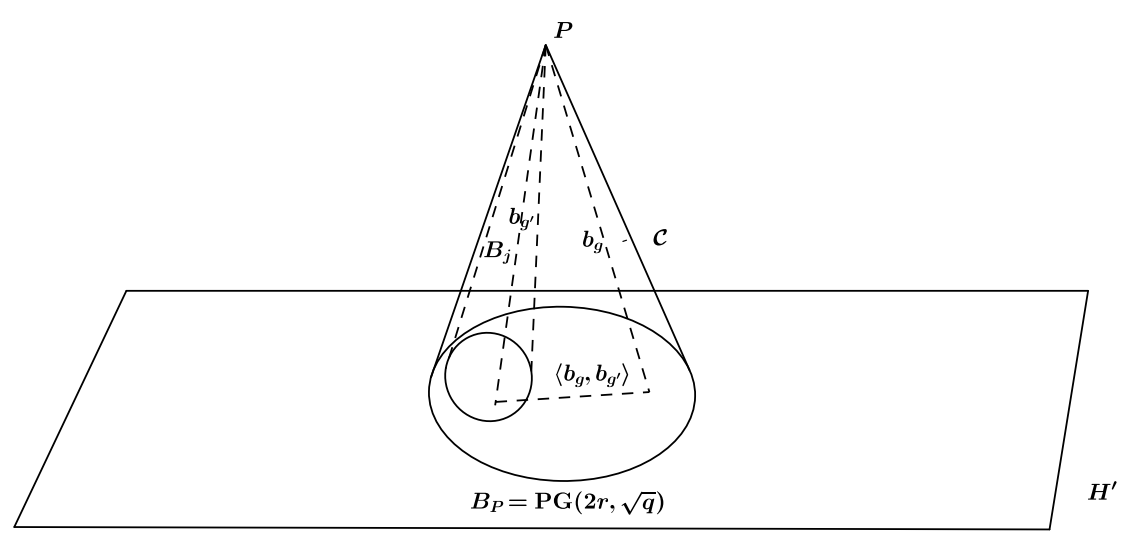

Figure 5. The setting of Lemma 2.13

since every hyperplane $H_{l^{\prime}}$ of $\mathcal{C}$, with $B_{l^{\prime}} \nsubseteq \mathcal{C}$, contains at least $q^{r}-\sqrt{q}$ holes not belonging to the cone $\mathcal{C}$. Taking into consideration inequalities (9), (12), (13), and (14), we obtain the following inequality:

$$
i \theta_{r-1} \frac{2 \sqrt{q}-1}{\sqrt{q}-1} \geq \theta_{r-1}(q+2 \sqrt{q})+\sqrt{q}+1 .
$$

This implies that for $r \geq 1$, necessarily $i \geq(q-1) / 2$; a contradiction. Therefore, $B_{j} \subseteq \mathcal{C}, \forall j \in\left\{1, \ldots, \theta_{2 r, \sqrt{q}}\right\}$.

We now arrive at the proof of one of the main results of this section. If the tight set $\mathcal{T}$ does not contain any $r$-space, then every hole $P$ belongs to a $(2 r+1)$-dimensional Baer subgeometry which consists entirely of holes.

Lemma 2.13. Let $\mathcal{T}$ be an $i$-tight set in $\mathcal{P}(2 r+1, q), 81 \leq q$ odd square, containing no $r$-spaces, $i<\left(q^{2 / 3}-1\right) / 2$. Then every hole belongs to a $(2 r+1)$-dimensional Baer subgeometry which consists entirely of holes, naturally embedded in $\mathrm{PG}(2 r+1, q)$.

Proof. Figure 5 shows the setting of Lemma 2.13.

Let $P$ be a hole and let $\mathcal{C}$ be the Baer cone with vertex $P$ defined by the rich hyperplanes through $P$. Denote by $H_{j}, j=1, \ldots, \theta_{2 r, \sqrt{q}}$, 
the hyperplanes of $\mathcal{C}$. Every hyperplane $H_{j}$ shares a unique $(2 r)$ dimensional Baer subgeometry $B_{j}$ with $\mathcal{T}, B_{j}$ is contained in $\mathcal{C}$, and $P$ is a point of $B_{j}$ (Corollary 2.8, Lemma 2.12, Lemma 2.11).

If a line of $\mathcal{C}$ belongs to a hyperplane $H_{j}$, then it intersects $B_{j}$ in a unique Baer subline (Lemma 2.12). We call a line of the cone $\mathcal{C}$ good if it contains exactly one Baer subline of holes. A line of the cone $\mathcal{C}$ that contains more than one Baer subline of holes, we call a bad line. The unique Baer subline of holes on a good line $g$ will be denoted by $b_{g}$.

Consider a hyperplane $H_{j}$ of the cone $\mathcal{C}$ and a good line $g$ outside of $H_{j}$. Then $\mathcal{D}:=\left\langle b_{g}, B_{j}\right\rangle$ is a $(2 r+1)$-dimensional Baer subgeometry. Obviously $P \in \mathcal{D}$, and the arguments which follow shall show that $\mathcal{D} \subseteq \mathcal{C}$.

Denote by $\gamma$ the number of good lines through $P$ sharing completely their unique Baer subline of holes with $\mathcal{D}$.

Suppose there exists a point $R \in \mathcal{D} \backslash \mathcal{T}$. Then each of the $\theta_{2 r-1, \sqrt{q}}$ Baer subplanes in $\mathcal{D}$ through the line $P R$ intersects at most one good line in its unique Baer subline of holes, and therefore

$$
\gamma \leq \theta_{2 r-1, \sqrt{q}}
$$

The proof of this assertion proceeds as follows. Let $\Delta$ denote a Baer subplane in $\mathcal{D}$ through the line $P R$ and suppose that $\Delta$ intersects at least two good lines $g^{\prime}$ and $g^{\prime \prime}$ through $P$ in their unique Baer subline of holes. The lines $g^{\prime}$ and $g^{\prime \prime}$ intersect the base $B_{P}$ of $\mathcal{C}$ in two points. These two points belong to $\theta_{2 r-2, \sqrt{q}}(2 r-1)$-dimensional Baer subgeometries that are the intersection of the base $B_{P}$ with some hyperplane of $\mathcal{C}$; denote one such hyperplane by $H_{j^{\prime}}$. Obviously, $g^{\prime}, g^{\prime \prime} \subseteq H_{j^{\prime}}$ which implies that $\left\langle b_{g^{\prime}}, b_{g^{\prime \prime}}\right\rangle \subseteq B_{j^{\prime}}$ since $g^{\prime}$ and $g^{\prime \prime}$ are good lines. Since $R \in\left\langle b_{g^{\prime}}, b_{g^{\prime \prime}}\right\rangle$, it follows that $R \in \mathcal{T}$; a contradiction.

Two distinct Baer sublines intersect in at most two points, so a bad line in $H_{j}$ contains at least $\sqrt{q}-1$ holes other than the ones shared with $B_{j}$. Consequently, if a hyperplane $H_{j}$ of $\mathcal{C}$ has $\beta_{j}$ bad lines, then

$$
\left|B_{j}\right|+\beta_{j}(\sqrt{q}-1) \leq q^{r}+i \theta_{r-1} .
$$

and

$$
\beta_{j} \leq \theta_{2 r-1, \sqrt{q}} \frac{q^{2 / 3}-2 \sqrt{q}-3}{2(q-1)} .
$$

Thus, since $H_{j}$ has $\theta_{2 r-1, \sqrt{q}}$ lines of $\mathcal{C}, H_{j}$ has at least $\theta_{2 r-1, \sqrt{q}}(1-$ $\left.\frac{q^{2 / 3}-2 \sqrt{q}-3}{2(q-1)}\right)$ good lines, and each good line $g^{\prime}$ defines, together with $g$, a Baer subplane $\left\langle b_{g}, b_{g^{\prime}}\right\rangle$ of holes in $\mathcal{D}$ and, by the arguments of the preceding paragraphs, also in $\mathcal{C}$. Each such Baer subplane $\left\langle b_{g}, b_{g^{\prime}}\right\rangle$ 
intersects $\sqrt{q}+1$ lines of the Baer cone $\mathcal{C}$ in a Baer subline. These subplanes mutually only share $b_{g}$, so in total there are at least

$$
\sqrt{q} \theta_{2 r-1, \sqrt{q}}\left(1-\frac{q^{2 / 3}-2 \sqrt{q}-3}{2(q-1)}\right)+1
$$

lines of $\mathcal{C}$ which intersect $\mathcal{D}$ in a Baer subline of holes.

Taking into consideration the bound (16) on $\beta_{j}$, the double counting of the set

$$
\{(\ell, H) \mid \ell \text { is a bad line, } H \text { is a hyperplane of } \mathcal{C}, \ell \subseteq H\}
$$

yields the following upper bound on the number of bad lines in $\mathcal{C}$ :

$$
\theta_{2 r, \sqrt{q}} \frac{q^{2 / 3}-2 \sqrt{q}-3}{2(q-1)},
$$

since the cone $\mathcal{C}$ has $\theta_{2 r, \sqrt{q}}$ hyperplanes and each line $\ell$ of the cone $\mathcal{C}$ belongs to $\theta_{2 r-1, \sqrt{q}}$ hyperplanes of the cone $\mathcal{C}$.

Subtracting it from (17) we get that

$$
\gamma \geq \theta_{2 r-1, \sqrt{q}} \frac{q^{3 / 2}-q^{7 / 6}+2 q+2 \sqrt{q}}{q-1}
$$

Now, inequalities (15) and (18) imply that

$$
\frac{q^{3 / 2}-q^{7 / 6}+2 q+2 \sqrt{q}}{q-1} \leq 1
$$

a contradiction.

The conclusion is that there is no point $R \in \mathcal{D} \backslash \mathcal{T}$, so the $(2 r+1)$ dimensional Baer subgeometry $\mathcal{D}$ through $P$ is completely contained in $\mathcal{T}$.

This $(2 r+1)$-dimensional Baer subgeometry $\mathcal{D}$ through $P$ is naturally embedded in $\mathrm{PG}(2 r+1, q)$ since the point $P$ belongs to $\theta_{2 r, \sqrt{q}}$ (rich) hyperplanes $H$ intersecting $\mathcal{D}$ in a $(2 r)$-dimensional Baer subgeometry of holes, naturally embedded in this hyperplane $H$.

The following theorem is the main theorem of this section.

Theorem 2.14. Let $\mathcal{T}$ be an i-tight set in $\mathcal{P}(2 r+1, q), 81 \leq q$ odd square, containing no $r$-spaces, $i<\left(q^{2 / 3}-1\right) / 2$. Then $\mathcal{T}$ is a union of pairwise disjoint $(2 r+1)$-dimensional Baer subgeometries, naturally embedded in $\mathrm{PG}(2 r+1, q)$.

Proof. It was shown in the previous lemma that every hole $P$ belongs to a $(2 r+1)$-dimensional Baer subgeometry of holes. It remains to be proven that this $(2 r+1)$-dimensional Baer subgeometry of holes through $P$ is unique. 
Consider two distinct $(2 r+1)$-dimensional Baer subgeometries $\mathcal{D}_{1}$ and $\mathcal{D}_{2}$ completely consisting of holes. Assume they share a hole. Then, dually, there exists a hyperplane $H$ that intersects $\mathcal{D}_{1}$ and $\mathcal{D}_{2}$ in respective $(2 r)$-dimensional Baer subgeometries $B_{H_{1}}$ and $B_{H_{2}}$ [10]. Obviously, $H$ is a rich hyperplane.

First we show that the hyperplane $H$ intersects $B_{H_{1}}$ and $B_{H_{2}}$ in the same $(2 r)$-dimensional Baer subgeometry. Assume the opposite. Then these two Baer subgeometries $B_{H_{1}}$ and $B_{H_{2}}$ share at most $\theta_{2 r-1, \sqrt{q}}+1$ points $[16,30]$ and

$$
|H \cap \mathcal{T}| \geq\left|B_{H_{1}} \cup B_{H_{2}}\right|>q^{r}+i \theta_{r-1},
$$

a contradiction. Thus, the hyperplane $H$ intersects $\mathcal{D}_{1}$ and $\mathcal{D}_{2}$ in the same $(2 r)$-dimensional Baer subgeometry $B_{H}$ of holes (Corollary 2.8) and $B_{H} \subseteq \mathcal{D}_{1} \cap \mathcal{D}_{2}[10]$. Hence, since the number of points of $\mathcal{D}_{1} \cap \mathcal{D}_{2}$ equals the number of common hyperplanes of $\mathcal{D}_{1}$ and $\mathcal{D}_{2}[9]$, there exists a second rich hyperplane $H^{\prime}$ that intersects $\mathcal{D}_{1}$ and $\mathcal{D}_{2}$ in the same $(2 r)$-dimensional Baer subgeometry $B_{H^{\prime}}$. However, distinct $(2 r+1)$ dimensional Baer subgeometries $\mathcal{D}_{1}$ and $\mathcal{D}_{2}$ share at most $\theta_{2 r, \sqrt{q}}+1$ points $[16,30]$, and since

$$
\left|\mathcal{D}_{1} \cap \mathcal{D}_{2}\right| \geq\left|B_{H} \cup B_{H^{\prime}}\right|>\theta_{2 r, \sqrt{q}}+1,
$$

we get a contradiction. Thus, it follows that $\mathcal{D}_{1} \cap \mathcal{D}_{2}=\emptyset$.

So far we have assumed that the tight set $\mathcal{T}$ of $\mathcal{P}(2 r+1, q)$ does not contain any $r$-spaces of $\mathrm{PG}(2 r+1, q)$. We only used common properties of the Hermitian variety $H(2 r+1, q)$ and of the symplectic polar space $W(2 r+1, q)$ to show that tight sets, not containing $r$-spaces, are unions of pairwise disjoint $(2 r+1)$-dimensional Baer subgeometries. If we assume that the tight sets $\mathcal{T}$ contain $r$-spaces of $\mathrm{PG}(2 r+1, q)$, then these two polar spaces $H(2 r+1, q)$ and $W(2 r+1, q)$ need to be discussed separately. This distinction arises from the fact that every $r$-space of $\mathrm{PG}(2 r+1, q)$ contained in the Hermitian variety $H(2 r+1, q)$ is a generator of $H(2 r+1, q)$, whereas for the symplectic space $W(2 r+1, q)$, this is not true.

In the following two subsections, we will also rely on the following result for obtaining the complete description of the investigated tight sets $\mathcal{T}$ in $\mathcal{P}(2 r+1, q)$.

Lemma 2.15. Let $\mathcal{T}_{1}$ and $\mathcal{T}_{2}$ be respectively $i_{1}$ - and $i_{2}$-tight sets of the finite classical polar space $\mathcal{P}(2 r+1, q)$, with $\mathcal{T}_{2} \subseteq \mathcal{T}_{1}$. Then $\mathcal{T}_{1} \backslash \mathcal{T}_{2}$ is an $\left(i_{1}-i_{2}\right)$-tight set of $\mathcal{P}(2 r+1, q)$. 
2.2. Tight sets in the Hermitian variety $H(2 r+1, q)$. To simplify the statements of this subsection, we first state a particular property of $(2 r+1)$-dimensional Baer subgeometries contained in a Hermitian variety $H(2 r+1, q)$ of $\mathrm{PG}(2 r+1, q), q$ square.

Lemma 2.16. Let $H(2 r+1, q)$ be a non-degenerate Hermitian variety of $P G(2 r+1, q)$, q square.

Let $\Sigma$ be a $(2 r+1)$-dimensional Baer subgeometry completely embedded in a Hermitian variety $H(2 r+1, q)$, then the Hermitian polarity of $H(2 r+1, q)$ induces a symplectic polarity in $\Sigma$.

Proof. Let $\ell$ be a line of $\mathrm{PG}(2 r+1, q)$ containing $\sqrt{q}+1$ points of $\Sigma$. Then, since $\Sigma \subset H(2 r+1, q)$, either $\ell \subset H(2 r+1, q)$ or $\mid \ell \cap H(2 r+$ $1, q) \mid=\sqrt{q}+1$, i.e., $\ell \cap H(2 r+1, q)=\ell \cap \Sigma$ or $\ell \subset H(2 r+1, q)$.

As a consequence, we have that, if $\tau$ is the non-linear involution of $\mathrm{PG}(2 r+1, q)$ fixing pointwise $\Sigma$, then $\tau$ fixes $H(2 r+1, q)$. For, this is proven in the following way. Take a point $P \in H(2 r+1, q) \backslash \Sigma$, then $P P^{\tau}$ is the unique line $\ell$ of $\Sigma$ through $P$. This line $\ell$ already contains the $\sqrt{q}+1$ points of $\ell \cap \Sigma$, and the point $P$ of $H(2 r+1, q) \backslash \Sigma$. Hence, this line $\ell=P P^{\tau} \subset H(2 r+1, q)$.

This proves that the non-linear involution $\tau$ fixes the Hermitian variety $H(2 r+1, q)$. By $[11$, Theorem 1.2, Cases 4$)$ and 6$)]$, it now follows that the Hermitian polarity of $H(2 r+1, q)$ induces a symplectic polarity in $\Sigma$.

Theorem 2.17. Let $\mathcal{T}$ be an $i$-tight set in the Hermitian variety $H(2 r+$ $1, q), 81 \leq q$ odd square, $i<\left(q^{2 / 3}-1\right) / 2$. Then $\mathcal{T}$ is a union of pairwise disjoint generators of $H(2 r+1, q)$ and $(2 r+1)$-dimensional Baer subgeometries.

Proof. Consider an $r$-space $\Delta$ contained in the tight set $\mathcal{T}$. It is known that this $r$-space $\Delta$ is a 1-tight set in the Hermitian variety $H(2 r+1, q)$. Then Lemma 2.15 shows that $\mathcal{T}^{\prime}:=\mathcal{T} \backslash \Delta$ is an $(i-1)$-tight set of $H(2 r+1, q)$.

Hence, removing all $r$-spaces from $\mathcal{T}$, we obtain a tight set of $H(2 r+$ $1, q)$ that is, by Theorem 2.14, a union of pairwise disjoint $(2 r+1)$ dimensional Baer subgeometries.

From Lemma 2.16, it follows that the Hermitian polarity of $H(2 r+$ $1, q)$ induces a symplectic polarity in these $(2 r+1)$-dimensional Baer subgeometries.

This concludes the proof for the case that $\mathcal{T}$ is an $i$-tight set in the Hermitian variety $H(2 r+1, q)$. Lemma 2.6 imposed the condition that $i<\left(q^{2 / 3}-1\right) / 2$. If $r \leq \sqrt{q}(q-2) /(18(q-1))$, then the next lemma 
can be used as an alternative to Lemma 2.6. Consequently, the upper bound on $i$ will be improved to $\left(q^{3 / 4}-1\right) / 2$ for the small values of $r$.

Lemma 2.18. Consider a point $P$ of the Hermitian variety $H(2 r+$ $1, q), q=p^{2 h}, 2<p$. Let $B$ be a non-trivial small minimal $r$-blocking set of the polar hyperplane $P^{\perp}$, with exponent $e$, contained in $H(2 r+$ $1, q)$. Let $|B|=q^{r}+\delta$. If $p^{e} \geq 18 r(q-1) /(q-2)$, then $e \mid h$.

Proof. Applying Corollary 1.11, we obtain that

$$
\delta \leq \frac{q-2}{8 r(q-1)} q^{r} .
$$

A line of $P^{\perp}$, that is not a line of $H(2 r+1, q)$, is either a tangent line or a $(\sqrt{q}+1)$-secant of $H(2 r+1, q)$ and then the intersection point set is isomorphic to a Baer subline. If a line is a $\left(p^{e}+1\right)$-secant of $B$, then the intersection point set is isomorphic to a subline $\mathrm{PG}\left(1, p^{e}\right)$ (Theorem 1.10). So, if a $(\sqrt{q}+1)$-secant of $H(2 r+1, q)$ is also a $\left(p^{e}+1\right)$-secant of $B$, necessarily $e \mid h$.

Assume now that every $\left(p^{e}+1\right)$-secant of $B$ is contained in a line of $H(2 r+1, q)$. We shall show that this assumption leads to the conclusion that $B$ is a trivial $r$-blocking set; a contradiction.

Let $\mathcal{E}$ be the set of all points of $B$ with exponent $e$. By Theorem 1.14,

$$
|\mathcal{E}| \geq q^{r}-3 \delta+2 p^{e}+4 \text {. }
$$

Let $R_{1} \in \mathcal{E}, R_{1} \neq P$, and let $\pi_{1}=\left\langle P, R_{1}\right\rangle=P R_{1}$. Obviously, $\pi_{1} \subseteq$ $H(2 r+1, q)$ since $\pi_{1} \subset P^{\perp}$. Denote by $\mathcal{E}_{1}$ the set of points of $B$ that belong to a $\left(p^{e}+1\right)$-secant of $B$ through $R_{1}$. Note that $\mathcal{E}_{1} \subseteq \mathcal{E}$. By Theorem 1.14,

$$
\left|\mathcal{E}_{1}\right| \geq\left(q^{r} / p^{e}-3(\delta-1) / p^{e}+2\right) p^{e}+1 .
$$

If $r=1$, then $\mathcal{E}_{1} \subseteq \pi_{1}$ since, by assumption, all $\left(p^{e}+1\right)$-secants of $B$ through $R_{1}$ lie on a line completely contained in $H(3, q)$, and $P R_{1}$ is the only line of $H(3, q)$ through $R_{1}$ lying in $P^{\perp}$. Both $\pi_{1}$ and $B$ are minimal blocking sets of $P^{\perp}$, and $B \cup \pi_{1}$ is a blocking set with

$$
\left|B \cup \pi_{1}\right| \leq|B|+\left|\pi_{1}\right|-\left|\mathcal{E}_{1}\right|<2 q .
$$

Therefore, $\pi_{1} \cup B$ is uniquely reducible to a minimal blocking set of $P^{\perp}$ (Theorem 1.2), so $B=\pi_{1}$. Since $B$ is a non-trivial blocking set, this is a contradiction.

If $r>1$, then $\left|\mathcal{E}_{1}\right|>\left|\pi_{1}\right|$. Let $R_{2} \in \mathcal{E}_{1} \backslash \pi_{1}$ and let $\pi_{2}=\left\langle P, R_{1}, R_{2}\right\rangle$. The line $R_{1} R_{2}$ is a $\left(p^{e}+1\right)$-secant of $B$, so by the assumption, it is contained in $H(2 r+1, q)$. The points $P, R_{1}$ and $R_{2}$ are not collinear and so $\pi_{2}$ is a 2 -space. The three lines $P R_{1}, P R_{2}$ and $R_{1} R_{2}$ are contained 
in $H(2 r+1, q)$ and do not share a common point, therefore $\pi_{2} \subseteq$ $H(2 r+1, q)$.

By $\mathcal{E}_{2}$, we denote the set of all points of $B$ that belong to a $\left(p^{e}+1\right)$ secant of $B$ through $R_{2}$. Then

$$
\left|\mathcal{E}_{1} \cap \mathcal{E}_{2}\right| \geq\left|\mathcal{E}_{1}\right|+\left|\mathcal{E}_{2}\right|-|B| .
$$

If $r>2$, then $\left|\mathcal{E}_{1} \cap \mathcal{E}_{2}\right|>\left|\pi_{2}\right|$. Similarly, for every $j=2, \ldots, r-1$, we obtain points $R_{j+1} \in\left(\mathcal{E}_{1} \cap \cdots \cap \mathcal{E}_{j}\right) \backslash \pi_{j}$, where $\pi_{j}=\left\langle P, R_{1}, \ldots, R_{j}\right\rangle$. By induction on the dimension $j$ of $\pi_{j},(j-1)$-spaces generated by $j$ points from the set $\left\{P, R_{1}, \ldots, R_{j}\right\}$ are contained in $H(2 r+1, q)$ and do not share a common $(j-2)$-space, therefore $\pi_{j} \subseteq H(2 r+1, q)$. By $\mathcal{E}_{j+1}$, we denote the set of all points of $B$ that belong to a $\left(p^{e}+1\right)$-secant of $B$ through $R_{j+1}$. Then

$$
\left|\mathcal{E}_{1} \cap \cdots \cap \mathcal{E}_{j+1}\right| \geq(j+1)\left(q^{r}-3(\delta-1)+2 p^{e}\right)-j\left(q^{r}+\delta\right)
$$

and that $\left|\mathcal{E}_{1} \cap \cdots \cap \mathcal{E}_{j+1}\right|>\left|\pi_{j+1}\right|$, when $j+1<r$.

In this way, we obtain an $r$-space $\pi_{r}=\left\langle P, R_{1}, \ldots, R_{r}\right\rangle$. By induction on the dimension $j$ of $\pi_{j}, \pi_{r} \subseteq H(2 r+1, q), \pi_{r}$ is a generator of $H(2 r+1, q)$. Note that $\pi_{r}$ cannot be extended to an $(r+1)$-space contained in $H(2 r+1, q)$.

The union $\pi_{r} \cup B$ is an $r$-blocking set of $P^{\perp}$. All $\left(p^{e}+1\right)$-secants of $B$ through $P$ and $R_{j}, j=1, \ldots, r$, are contained in $H(2 r+1, q)$, so

$$
\left|\pi_{r} \cap B\right| \geq\left|\mathcal{E}_{1}\right|+\cdots+\left|\mathcal{E}_{r}\right|-(r-1)|B| \geq q^{r}-\delta(4 r-1)+2 r p^{e} .
$$

Applying (19),

$$
\left|\pi_{r} \cup B\right|=\left|\pi_{r}\right|+|B|-\left|\pi_{r} \cap B\right|<2 q^{r},
$$

and so $\pi_{r} \cup B$ is uniquely reducible to a minimal $r$-blocking set of $P^{\perp}$ (Theorem 1.2). Both $\pi_{r}$ and $B$ are minimal $r$-blocking sets in $\pi_{r} \cup B$, therefore $B=\pi_{r}$. Since $B$ is a non-trivial $r$-blocking set of $P^{\perp}$, this is a contradiction.

Thus, at least one $\left(p^{e}+1\right)$-secant of $B$ is contained in a $(\sqrt{q}+1)$ secant of $H(2 r+1, q)$ and $e \mid h$.

Lemma 2.19. Consider a point $P$ of the Hermitian variety $H(2 r+$ $1, q), q=p^{2 h}, 2<p$ prime, $h \geq 1$. Let $B$ be a non-trivial small minimal $r$-blocking set of the polar hyperplane $P^{\perp}$, with exponent $e$, contained in $H(2 r+1, q)$. Let $|B|=q^{r}+\delta$. If $p^{e} \geq 18 r(q-1) /(q-2)$ and $|B|<q^{r}+q^{r-1 / 4} / 2+1$, then $e=h$.

The proof of this lemma is similar to the proof of Lemma 2.6.

If we assume that $r \leq p(q-2) /(18(q-1))$ and $i<\left(q^{3 / 4}-1\right) / 2$, then the preceding lemma is valid for all exponents $e$, since $e \geq 1$ (Theorem 
1.10). It is easy to adjust the calculations in the proofs of Section 2.1 to obtain the following result.

Theorem 2.20. Let $\mathcal{T}$ be an $i$-tight set in the Hermitian variety $H(2 r+$ $1, q), 81 \leq q, q=p^{2 h}, 2<p$ prime, $h \geq 1, r \leq p(q-2) /(18(q-1))$ and $i<\left(q^{3 / 4}-1\right) / 2$. Then $\mathcal{T}$ is a union of pairwise disjoint generators of $H(2 r+1, q)$ and $(2 r+1)$-dimensional Baer subgeometries.

For small $r$, this theorem is better than Theorem 2.17, but as the conditions of this theorem state, it cannot be used for large values of $r$.

2.3. Tight sets in the symplectic polar space $W(2 r+1, q)$. The symplectic polar space $W(2 r+1, q)$ sometimes contains particular 2tight sets which are the union $\Delta \cup \Delta^{\perp}$, for $\Delta$ an $r$-space of $\mathrm{PG}(2 r+1, q)$, satisfying $\Delta \cap \Delta^{\perp}=\emptyset$. This only occurs for $r$ odd, as will be proven in Theorem 2.23.

To simplify the proofs, we first prove that such a union $\Delta \cup \Delta^{\perp}$, for $\Delta$ an $r$-space of $\mathrm{PG}(2 r+1, q)$, satisfying $\Delta \cap \Delta^{\perp}=\emptyset$, indeed is a 2 -tight set of $W(2 r+1, q)$.

Lemma 2.21. Suppose that for the polarity $\perp$ of a symplectic polar space $W(2 r+1, q)$, there exists an $r$-space $\Delta$ satisfying $\Delta \cap \Delta^{\perp}=\emptyset$. Then $\mathcal{T}^{\prime}=\Delta \cup \Delta^{\perp}$ is a 2-tight set of $W(2 r+1, q)$.

Proof. Let $P \in W(2 r+1, q)$. Obviously,

$$
\left|P^{\perp} \cap \mathcal{T}^{\prime}\right|=\left|P^{\perp} \cap \Delta\right|+\left|P^{\perp} \cap \Delta^{\perp}\right| \text {. }
$$

Assume first that $P \notin \mathcal{T}^{\prime}$. Then $P \notin \Delta \cup \Delta^{\perp}$ and $\Delta, \Delta^{\perp} \nsubseteq P^{\perp}$. Thus, both $\Delta$ and $\Delta^{\perp}$ intersect $P^{\perp}$ in an $(r-1)$-space.

If $P \in \mathcal{T}^{\prime}$, without loss of generality, assume that $P \in \Delta$ and $P \notin$ $\Delta^{\perp}$. Then $\Delta^{\perp} \subseteq P^{\perp}, \Delta \nsubseteq P^{\perp}$, and so $\Delta$ intersects $P^{\perp}$ in an $(r-1)$ space.

Applying these observations, it follows that

$$
\left|P^{\perp} \cap \mathcal{T}^{\prime}\right|= \begin{cases}2 \theta_{r-1}+q^{r} & , P \in \mathcal{T}^{\prime}, \\ 2 \theta_{r-1} & , P \notin \mathcal{T}^{\prime},\end{cases}
$$

and $\mathcal{T}^{\prime}$ is a 2-tight set of $W(2 r+1, q)$.

Lemma 2.22. Let $\mathcal{T}$ be an $i$-tight set in the symplectic polar space $W(2 r+1, q), 81 \leq q$ odd square, $i<\left(q^{2 / 3}-1\right) / 2$. Then $\mathcal{T}$ is a union of pairwise disjoint generators of $W(2 r+1, q)$, pairs of disjoint $r$-spaces $\left\{\Delta, \Delta^{\perp}\right\},(2 r+1)$-dimensional Baer subgeometries invariant under the symplectic polarity of $W(2 r+1, q)$, and pairs of disjoint $(2 r+1)$-dimensional Baer subgeometries $\left\{\Omega_{1}, \Omega_{2}\right\}$ which correspond to each other under the symplectic polarity of $W(2 r+1, q)$. 
Proof. Denote by $\Delta$ an $r$-space contained in $\mathcal{T}$. If $\Delta$ is a generator of $W(2 r+1, q)$, then $\Delta$ is a 1-tight set of $W(2 r+1, q)$ and then $\mathcal{T} \backslash \Delta$ is an $(i-1)$-tight set of $W(2 r+1, q)$ (Lemma 2.15).

Assume now that $\Delta$ is not a generator of $W(2 r+1, q)$. Then $\Delta \neq \Delta^{\perp}$. We shall then show that $\mathcal{T}^{\prime}:=\mathcal{T} \backslash\left(\Delta \cup \Delta^{\perp}\right)$ is an $(i-2)$-tight set of $W(2 r+1, q)$. First we show that $\Delta^{\perp} \subseteq \mathcal{T}$ and that $\Delta \cap \Delta^{\perp}=\emptyset$.

If $H$ is a hyperplane through $\Delta$, then $H$ is a rich hyperplane. Thus, there exists a unique point $Q \in \mathcal{T}$ such that $H=Q^{\perp}$. Then, $\Delta \subseteq Q^{\perp}$, which implies that $Q \in \Delta^{\perp}$. There are $\theta_{r}$ hyperplanes through $\Delta$ and $\theta_{r}$ points in $\Delta^{\perp}$, hence every point of $\Delta^{\perp}$ is a hole.

Assume now that $\Delta \cap \Delta^{\perp} \neq \emptyset$ and let $R \in \Delta \cap \Delta^{\perp}$. Then, $\Delta \cup \Delta^{\perp} \subseteq$ $R^{\perp}$. Two distinct $r$-spaces can intersect in at most an $(r-1)$-space, thus

$$
\left|\Delta \cup \Delta^{\perp}\right| \geq 2 \theta_{r}-\theta_{r-1}>\left|R^{\perp} \cap \mathcal{T}\right|
$$

a contradiction. Therefore, $\Delta$ and $\Delta^{\perp}$ are disjoint.

From Lemma 2.21, $\Delta \cup \Delta^{\perp}$ is a 2-tight set of $W(2 r+1, q)$, and, by Lemma $2.15, \mathcal{T} \backslash\left(\Delta \cup \Delta^{\perp}\right)$ is an $(i-2)$-tight set of $W(2 r+1, q)$.

In both cases, whether $\Delta$ is a generator of $W(2 r+1, q)$ or not, when removing $\Delta \cup \Delta^{\perp}$ from $\mathcal{T}$, the structure of a tight set of $W(2 r+1, q)$ is preserved. So, we can assume that $\mathcal{T}$ does not contain any $r$-spaces. Then, by Theorem 2.14, $\mathcal{T}$ is a union of pairwise disjoint $(2 r+1)$ dimensional Baer subgeometries.

It follows from [13, Lemma 3.11] that these $(2 r+1)$-dimensional Baer subgeometries, contained in $\mathcal{T}$, either are invariant under the symplectic polarity of $W(2 r+1, q)$ or they come in pairs of disjoint $(2 r+$ 1)-dimensional Baer subgeometries $\left\{\Omega_{1}, \Omega_{2}\right\}$ which correspond to each other under the symplectic polarity of $W(2 r+1, q)$, meaning that for every point $P$ of $\Omega_{1}, P^{\perp} \cap \Omega_{2}$ is a $2 n$-dimensional Baer subgeometry, and for every point $P$ of $\Omega_{2}, P^{\perp} \cap \Omega_{1}$ is a $2 n$-dimensional Baer subgeometry.

But if $r$ is even, this result can be improved, since for $r$ even, $W(2 r+$ $1, q)$ does not contain pairs of disjoint $r$-spaces $\left\{\Delta, \Delta^{\perp}\right\}$.

Theorem 2.23. If $r$ is even, then in the symplectic polar space $W(2 r+$ $1, q)$, there exist no pairs of disjoint $r$-spaces $\left\{\Delta, \Delta^{\perp}\right\}$ of $\mathrm{PG}(2 r+1, q)$.

Proof. Assume that there exists a pair $\left\{\Delta, \Delta^{\perp}\right\}$ of disjoint $r$-spaces in the symplectic polar space $W(2 r+1, q)$. Then, no point $P \in \Delta$ is collinear with all the points of $\Delta$ in $W(2 r+1, q)$. For, the points of $\Delta$ form in $W(2 r+1, q)$ a symplectic polar space with singular space $\Delta \cap \Delta^{\perp}$. Since $\Delta$ and $\Delta^{\perp}$ are disjoint, the points of $\Delta$ necessarily form 
in $W(2 r+1, q)$ a non-singular symplectic polar space of dimension $r$; a contradiction since $r$ is even.

If $r$ is odd, the previous theorem is not valid. Consider in $\mathrm{PG}(2 r+$ $1, q), r$ odd, an $r$-space $\Delta$ such that $\Delta \cap W(2 r+1, q)$ is a symplectic polar space $W(r, q)$, then $\Delta \cap \Delta^{\perp}=\emptyset$. So, by Lemma $2.21, \mathcal{T}=\Delta \cup \Delta^{\perp}$ is a 2-tight set of $W(2 r+1, q), r$ odd.

Now, we can present the improvement of Theorem 2.22.

Corollary 2.24. Let $\mathcal{T}$ be an $i$-tight set in the symplectic polar space $W(2 r+1, q), 81 \leq q$ odd,$i<\left(q^{2 / 3}-1\right) / 2$.

(1) If $r$ is odd, then $\mathcal{T}$ is a union of pairwise disjoint generators of $W(2 r+1, q)$, pairs of disjoint $r$-spaces $\left\{\Delta, \Delta^{\perp}\right\},(2 r+1)$ dimensional Baer subgeometries invariant under the symplectic polarity, and pairs of disjoint $(2 r+1)$-dimensional Baer subgeometries $\left\{\Omega_{1}, \Omega_{2}\right\}$ which correspond to each other under the symplectic polarity.

(2) If $r$ is even, then $\mathcal{T}$ is a union of pairwise disjoint generators of $W(2 r+1, q),(2 r+1)$-dimensional Baer subgeometries invariant under the symplectic polarity, and pairs of disjoint $(2 r+1)$ dimensional Baer subgeometries $\left\{\Omega_{1}, \Omega_{2}\right\}$ which correspond to each other under the symplectic polarity.

Remark 2.25. In the preceding characterization theorem, the possibility of a pair of disjoint $(2 r+1)$-dimensional Baer subgeometries $\left\{\Omega_{1}, \Omega_{2}\right\}$ which correspond to each other under the symplectic polarity of $W(2 r+1, q)$ is mentioned.

This possibility occurs because such a pair would constitute a $(2 \sqrt{q}+$ 2)-tight set in $W(2 r+1, q)$.

For $W(3,4)$, the non-existence of such a pair has been proven $[13$, Remark 3.13].

For $W(3,9)$, the existence of such a pair has been found. We thank the referee for presenting us with this example.

Theorem 2.26. The symplectic polar space $W(3,9)$ contains an 8tight set $\mathcal{T}$ which is a pair of disjoint 3-dimensional Baer subgeometries $\left\{\Omega_{1}, \Omega_{2}\right\}$ which correspond to each other under the symplectic polarity $\perp$ of $W(3,9)$.

Proof. In $W(3,9)$, it is possible to find two disjoint Baer subgeometries PG $(3,3)$ having the correct properties. With the aid of a computer, it is possible to check that the following configuration occurs in $W(3,9)$.

Consider a Baer subgeometry $\mathrm{PG}(3,3)=\Sigma$ such that there are exactly 10 (totally isotropic) lines of $W(3,9)$ that meet $\Sigma$ in a Baer subline. Moreover, this set $\mathcal{S}$ of 10 lines induces a regular spread in $\Sigma$. 
Then, the lines of $\mathcal{S}$ form a pseudoregulus, i.e., the set of points of $\mathrm{PG}(3,9)$ covered by the 10 lines of $\mathcal{S}$ is covered by the trivially intersecting sets formed by 2 transversal lines and 2 subgeometries, one of which is $\Sigma$.

Let $\Sigma^{\prime}$ be the other 3-dimensional Baer subgeometry. Then, if $P \in \Sigma$, then $P^{\perp} \cap \Sigma^{\prime}$ is a Baer subplane, and vice versa, as required.

In the preceding example, if $G$ denotes the stabilizer of the Baer subgeometry $\Sigma$ in the symplectic group $P S p(4,9)$ fixing $W(3,9)$, then $|G|=4\left|A_{6}\right|$.

3. MAXimal PARTial SPREAds IN THE SYMPleCtiC POlAR SPACE $W(2 r+1, q)$ AND IN THE HERMitian POLAR SPACE $H(2 r+1, q)$

In this section we apply the obtained characterization results on tight sets in the symplectic polar spaces $W(2 r+1, q)$ and $H(2 r+1, q)$ to maximal partial spreads in $W(2 r+1, q)$ and in $H(2 r+1, q)$. The application of the result on tight sets, then yields an extension of the results from [20] on the bounds on the sizes of maximal partial spreads in $W(2 r+1, q)$. In [20], the technique was based on a link between minihypers and partial spreads. To obtain improved results, we show that the set of holes of a partial spread of deficiency $\delta$ in the finite classical polar spaces $W(2 r+1, q), H(2 r+1, q)$, and $Q^{+}(2 r+1, q)$ is a $\delta$-tight set in these finite classical polar spaces.

A spread $\mathcal{S}$ of a finite classical polar space $\mathcal{P}$ is a set of generators that partitions the point set of $\mathcal{P}$. The cardinality of a spread $\mathcal{S}$ is then $|\mathcal{P}| / \theta_{r}$, when $r+1$ is the rank of $\mathcal{P}$.

Not all finite classical polar spaces contain spreads. We present here in Table 1 the Table 2 of [14] which gives the known results regarding (non-)existence of spreads in finite classical polar spaces. For the exact references to these results, we refer to the bibliography of [14].

A partial spread of $\mathcal{P}$ is a set of pairwise disjoint generators. It is called maximal when it is not contained in a larger partial spread. The cardinality of a partial spread $\mathcal{S}$ is $|\mathcal{P}| / \theta_{r}-\delta$ for some integer $\delta$ which is called the deficiency of $\mathcal{S}$. There are $\delta \theta_{r}$ points of $\mathcal{P}$ not covered by $\mathcal{S}$. Such points are called holes of $\mathcal{S}$.

The following theorem reveals the link between minihypers and partial spreads.

Theorem 3.1. [20] Let $\mathcal{P}$ be a classical polar space of rank $r+1$ in $\operatorname{PG}(n, q)$. If $\mathcal{S}$ is a partial spread of $\mathcal{P}$ with deficiency $\delta<q$, then the set of holes of $\mathcal{S}$ forms a $\left\{\delta \theta_{r}, \delta \theta_{r-1} ; n, q\right\}$-minihyper. 


\begin{tabular}{|c|l|}
\hline Space & Existence \\
\hline$Q^{-}(5, q)$ & Yes \\
\hline$Q^{-}(2 n+1, q)$ & $q$ even: Yes \\
\hline$Q(2 n, q)$ & $n \geq 2, q$ even: Yes \\
\hline$Q(6, q)$ & $\begin{array}{l}q \text { odd, } q \text { prime: Yes } \\
q \text { odd, } q \not \equiv 1 \bmod 3: \text { Yes }\end{array}$ \\
\hline$Q(4 n, q)$ & $q$ odd: No \\
\hline$Q^{+}(4 n+1, q)$ & No \\
\hline$Q^{+}(3, q)$ & Yes \\
\hline$Q^{+}(7, q)$ & $q$ odd, $q$ prime: Yes \\
& $q$ odd, $q \not \equiv 1 \bmod 3:$ Yes \\
\hline$Q^{+}(4 n+3, q)$ & $q$ even: Yes \\
\hline$W(2 n+1, q)$ & Yes \\
\hline$H\left(2 n+1, q^{2}\right)$ & No \\
\hline$H(4,4)$ & No \\
\hline
\end{tabular}

TABLE 1. Existence and non-existence results on spreads

In [20], this link was then used to obtain new bounds on the sizes of maximal partial spreads of finite classical polar spaces.

The following result gives a strong characterization result on minihypers, contained in the non-singular quadrics $Q(2 r, q)$ and $Q^{-}(2 r+1, q)$.

Theorem 3.2. [13, Theorem 2.10]

(1) An $\left\{i \theta_{r-1}, i \theta_{r-2} ; 2 r, q\right\}$-minihyper $F$ contained in $Q(2 r, q)$, with $i \leqslant q / 2-1$, consists of $i$ pairwise disjoint generators.

(2) An $\left\{i \theta_{r-1}, i \theta_{r-2} ; 2 r+1, q\right\}$-minihyper $F$ contained in $Q^{-}(2 r+$ $1, q)$, with $i \leqslant q / 2-1$, consists of $i$ pairwise disjoint generators.

For the hyperbolic quadrics $Q^{+}(2 r+1, q)$, there is a strong characterization theorem of Beukemann and Metsch, immediately for tight sets.

Corollary 3.3. [2, Theorem 1.1] Let $F$ be an i-tight set of $Q^{+}(2 r+1, q)$, with $i \leq q$ when $1 \leq r \leq 3$, and with $i \leq q-1$ for $r \geq 4, q \geq 71$, then $F$ is the union of $i$ mutually disjoint generators. If $r$ is even, then necessarily $i \leqslant 2$.

Theorem 3.2 immediately induces an extendability result on partial spreads of $Q(2 r, q)$ and $Q^{-}(2 r+1, q)$, having small positive deficiency $\delta$, to spreads, when spreads exist in the corresponding finite classical polar spaces. Otherwise they induce upper bounds on the sizes of partial spreads in these finite classical polar spaces. 
We address these extendability results in the next theorem. We immediately state also the corresponding result on the extendability of maximal partial spreads of the hyperbolic quadric $Q^{+}(2 r+1, q), r$ odd, which relies on Corollary 3.3 and Lemma 3.6 which follows. But we refer to Table 1 on the known (non-)existence results on spreads in finite classical polar spaces to check whether it is known if these finite classical polar spaces contain spreads.

Theorem 3.4. (1) Every partial spread of $Q(2 r, q)$, with deficiency $\delta$ satisfying $0<\delta \leqslant q / 2-1$, can be extended to a spread of $Q(2 r, q)$.

(2) Every partial spread of $Q^{-}(2 r+1, q)$, with deficiency $\delta$ satisfying $0<\delta \leqslant q / 2-1$, can be extended to a spread of $Q^{-}(2 r+1, q)$.

(2) Every partial spread of $Q^{+}(2 r+1, q), r$ odd, with deficiency $\delta$ satisfying $0<\delta \leq q$ when $1 \leq r \leq 3$, and with $0<\delta \leq q-1$ for $r \geq 4, q \geq 71$, can be extended to a spread of $Q^{+}(2 r+1, q)$.

In this section, we address improved results on the extendability of partial spreads in the symplectic polar spaces $W(2 r+1, q)$ and $H(2 r+$ $1, q)$, which rely on the improved results on tight sets of the preceding section. Because of the isomorphism between $Q(2 r+2, q), q$ even, and $W(2 r+1, q), q$ even, we focus on the case $q$ odd for the polar space $W(2 r+1, q)$.

The following result is the known result.

Theorem 3.5. [20] Let $q+\epsilon$ denote the size of the smallest non-trivial blocking sets in $\mathrm{PG}(2, q)$. Let $\mathcal{S}$ be a maximal partial spread of deficiency $\delta$ in $W(2 r+1, q), q$ odd. Suppose that either $\delta<\epsilon$, or $q>16$ is a square and $\delta<q^{5 / 8} / \sqrt{2}+1$. Then $\delta$ is even.

The following lemma will show that the set of holes of a maximal partial spread $\mathcal{S}$ of deficiency $\delta$ in $W(2 r+1, q), Q^{+}(2 r+1, q)$, or $H(2 r+$ $1, q)$ is a $\delta$-tight set in these finite classical polar spaces. The application of Theorem 2.24 for $W(2 r+1, q)$ raises in the previous result the upper bound on $\delta$ from $q^{5 / 8} / \sqrt{2}+1$ to $\left(q^{2 / 3}-1\right) / 2$. Consequently, new bounds on the sizes of maximal partial spreads in $W(2 r+1, q)$ are obtained.

Lemma 3.6. Let $\mathcal{S}$ be a partial spread with deficiency $\delta$ in the symplectic polar space $W(2 r+1, q)$, respectively $Q^{+}(2 r+1, q), H(2 r+1, q)$. Then the set of holes $\mathcal{T}$ of $\mathcal{S}$ is a $\delta$-tight set in $W(2 r+1, q)$, respectively $Q^{+}(2 r+1, q), H(2 r+1, q)$.

Proof. Let $\mathcal{P}$ be the symplectic polar space $W(2 r+1, q)$, respectively $Q^{+}(2 r+1, q), H(2 r+1, q)$. The set of all points of such a polar space $\mathcal{P}$ is a (trivial) $\mathcal{O}$-tight set in $\mathcal{P}$, where $\mathcal{O}=|\mathcal{P}| / \theta_{r}$. The partial spread 
$\mathcal{S}$ is a $(\mathcal{O}-\delta)$-tight set in $\mathcal{P}$. Hence, by Lemma 2.15, the set of holes $\mathcal{T}=\mathcal{P} \backslash \mathcal{S}$ is a $\delta$-tight set in $\mathcal{P}$.

Corollary 3.7. Let $\mathcal{S}$ be a maximal partial spread of deficiency $\delta$ in the symplectic polar space $W(2 r+1, q)$.

(1) Let $q+\epsilon$ denote the size of the smallest nontrivial blocking sets in $\mathrm{PG}(2, q)$. Assume $\delta<\epsilon$. Then:

(a) If $q$ is odd and $r$ is odd, then $\delta$ is even and the set of holes is the union of pairwise disjoint pairs of $r$-spaces $\left\{\Delta, \Delta^{\perp}\right\}$, with $\Delta \cap \Delta^{\perp}=\emptyset$.

(b) Otherwise, any partial $r$-spread of deficiency $\delta$ of $W(2 r+$ $1, q)$ can be extended to a spread of $W(2 r+1, q)$.

(2) Assume $81 \leq q$ is an odd square, $\delta<\left(q^{2 / 3}-1\right) / 2$.

(a) If $r$ is odd, then $\delta=s_{1}(\sqrt{q}+1)+s_{2}, s_{1}, s_{2}$ nonnegative integers, $s_{2}$ even, and the set of holes is the union of pairwise disjoint $(2 r+1)$-dimensional Baer subgeometries and of pairwise disjoint pairs of r-spaces $\left\{\Delta, \Delta^{\perp}\right\}$, with $\Delta \cap \Delta^{\perp}=\emptyset$.

(b) If $r$ is even, then $\delta \equiv 0(\bmod \sqrt{q}+1)$ and the set of holes is the union of pairwise disjoint $(2 r+1)$-dimensional Baer subgeometries.

Proof. The set of holes $\mathcal{T}$ of a maximal partial spread $\mathcal{S}$ of deficiency $\delta$ in the symplectic polar space $W(2 r+1, q)$ is a $\delta$-tight set (Lemma 3.6).

It is known that a 1-tight set is equal to a generator of $W(2 r+1, q)$ [17, Theorem 9.1].

Assume $2 \leq \delta<\epsilon$. Then, it is known that a $\delta$-tight set in $W(2 r+1, q)$ is equal to a $\left\{\delta \theta_{r}, \delta \theta_{r-1} ; 2 r+1, q\right\}$-minihyper (Lemma 2.2). If $q$ is odd and $r$ is odd, then, by Corollary 2.24, the set of holes $\mathcal{T}$ is a union of pairwise disjoint pairs of disjoint $r$-spaces $\left\{\Delta, \Delta^{\perp}\right\}$, implying that $\delta$ is even. Note that the possibility of the $(2 r+1)$-dimensional Baer subgeometries in the statement of Corollary 2.24 cannot occur here since $\delta<\epsilon$.

If $q$ is odd and $r$ is even, then, by Corollary 2.24, a $\delta$-tight set in $W(2 r+1, q)$ is a union of pairwise disjoint generators of $W(2 r+1, q)$, so $\mathcal{S}$ must be equal to a spread since the generators in $\mathcal{T}$ extend $\mathcal{S}$ to a spread. Finally, it was shown in [20] that when $q$ is even, any partial spread of $W(2 r+1, q)$, with deficiency $\delta<\epsilon$, can be extended to a spread in $W(2 r+1, q)$.

Assume $81 \leq q$ is an odd square, $\delta<\left(q^{2 / 3}-1\right) / 2$. Then we can apply Corollary 2.24. If $r$ is odd, then the set of holes of $\mathcal{S}$ is a union of pairwise disjoint $(2 r+1)$-dimensional Baer subgeometries and pairs 
of disjoint $r$-spaces $\left\{\Delta, \Delta^{\perp}\right\}$. Hence, $\delta=s_{1}(\sqrt{q}+1)+s_{2}$, where $s_{1}, s_{2}$ are nonnegative integers with $s_{2}$ even. If $r$ is even, then the set of holes of $\mathcal{S}$ is a union of pairwise disjoint $(2 r+1)$-dimensional Baer subgeometries.

Remark 3.8. The investigation of maximal partial spreads in $W(2 r+$ $1, q)$ is not only complicated because of the distinct property for $W(2 r+$ $1, q), r$ is even or odd; it also depends on the field $\mathbb{F}_{q}$.

In particular, the symplectic polar space $W(3, q)$ has maximal partial spreads of size $q^{2}-1$ when $q=3,5,7,11$ [15].

But, De Beule and Gács proved in [12] that $W(3, q), q$ an odd prime power different from a prime, does not have maximal partial spreads of size $q^{2}-1$.

We now conclude this article with the new extendability result on partial spreads on the Hermitian polar spaces $H(2 r+1, q)$, which relies on the characterization result on tight sets of Theorem 2.17. Note that the Hermitian polar space $H(2 r+1, q)$ does not have spreads (Table 1 ), so we immediately formulate this result as a result on the possible sizes of large maximal partial spreads.

Theorem 3.9. Let $\mathcal{S}$ be a maximal partial spread of the Hermitian variety $H(2 r+1, q), 81 \leq q$ odd square, with deficiency $\delta$ satisfying $0<\delta<\left(q^{2 / 3}-1\right) / 2$. Then $\delta \equiv 0(\bmod \sqrt{q}+1)$ and the set of holes is the union of $\delta /(\sqrt{q}+1)$ pairwise disjoint $(2 r+1)$-dimensional Baer subgeometries $\mathrm{PG}(2 r+1, \sqrt{q})$ in which the Hermitian polarity of $H(2 r+1, q)$ induces a symplectic polarity.

Acknowledgements: 1. Anamari Nakić is supported in part by a STSM grant from the COST project Random network coding and designs over $G F(q)$ (COST IC-1104) and in part by the Croatian Science Foundation under the project 1637.

2. The authors thank the referee for the many suggestions which improved the article. We especially wish to thank the referee for the proof of Lemma 2.16 and for giving us the example of two disjoint Baer subgeometries $\mathrm{PG}(3,3)$ in $\mathrm{PG}(3,9)$ which correspond to each other under a symplectic polarity $W(3,9)$ (Theorem 2.26 ), thus providing us with an example of a $(2 \sqrt{q}+2)$-tight set in $W(2 r+1, q)=W(3,9)$ which is the union of two disjoint Baer subgeometries $\mathrm{PG}(2 r+1, \sqrt{q})=$ $\mathrm{PG}(3,3)$.

\section{REFERENCES}

[1] J. Bamberg, S. Kelly, M. Law, T. Penttila, Tight sets and m-ovoids of finite polar spaces, J. Combin. Theory Ser. A 114 (2007), no. 7, 1293-1314. 
[2] L. Beukemann, K. Metsch, Small tight sets of hyperbolic quadrics, Des. Codes Cryptogr. 68 (2013), 11-24.

[3] A. Blokhuis, Blocking sets in Desarguesian planes, in: Paul Erdős is Eighty, vol. 2, eds. D. Miklós, V. T. Sós, T. Szőnyi, Bolyai Soc. Math. Studies (1996), $133-155$.

[4] A. Blokhuis, On the size of a blocking set in PG(2,p), Combinatorica 14 (1994), $273-276$.

[5] A. Blokhuis, L. Lovász, L. Storme, T. Szőnyi, On multiple blocking sets in Galois planes, Adv. Geom. 7 (2007), 39-53.

[6] A. Blokhuis, L. Storme, T. Szönyi, Lacunary polynomials, multiple blocking sets and Baer subplanes, J. London Math. Soc. (2) 60 (1999), 321-332.

[7] R. C. Bose, R. C. Burton, A characterization of flat spaces in a finite geometry and the uniqueness of the Hamming and the MacDonald code, J. Combin. Theory 1 (1966), 96-104.

[8] A. A. Bruen, Baer subplanes and blocking sets, Bull. Amer. Math. Soc. 76 (1970), 342-344.

[9] A. A. Bruen, Intersection of Baer subgeometries, Arch. Math. 39 (1982), no. $3,285-288$.

[10] A. A. Bruen, J. W. P. Hirschfeld, Intersections in projective space I: Combinatorics, Math. Z. 193 (1986), no. 2, 215-225.

[11] A. Cossidente, F. Pavese, On the geometry of unitary involutions, Finite Fields Appl. 36 (2015), 14-28.

[12] J. De Beule, A. Gács, Complete arcs on the parabolic quadric $Q(4, q)$, Finite Fields Appl. 14 (2008), 14-21.

[13] J. De Beule, P. Govaerts, A. Hallez, L. Storme, Tight sets, weighted m-covers, weighted m-ovoids, and minihypers, Des. Codes Cryptogr. 50 (2009), no. 2, 187-201.

[14] J. De Beule, A. Klein, K. Metsch, L. Storme, Partial ovoids and partial spreads of classical finite polar spaces, Serdica Math. J. 34 (2008), 689-714.

[15] S. De Winter, K. Thas, Bounds on partial ovoids and spreads in classical generalized quadrangles, Innov. Incidence Geom. 11 (2010), 19-33.

[16] G. Donati, N. Durante, On the intersection of two subgeometries of $\mathrm{PG}(n, q)$, Des. Codes Cryptogr. 46 (2008), no. 3, 261-267.

[17] K. Drudge, Extremal sets in projective and polar spaces, $\mathrm{PhD}$ thesis. The University of Western Ontario, 1998.

[18] V. Fack, Sz. L. Fancsali, L. Storme, G. Van de Voorde, J. Winne, Small weight codewords in the codes arising from Desarguesian projective planes, Des. Codes Cryptogr. 46 (2008), 25-43.

[19] P. Govaerts, L. Storme, On a particular class of minihypers and its applications. II. Improvements for q square, J. Combin. Theory Ser. A 97 (2002), no. 2, 369-393.

[20] P. Govaerts, L. Storme, H. Van Maldeghem, On a particular class of minihypers and its applications. III: Applications, Europ. J. Combin. 23 (2002), 659-672.

[21] A. Hallez, Linear codes and blocking structures in finite projective and polar spaces, $\mathrm{PhD}$ thesis, Ghent University (2010).

[22] N. Hamada, Characterization of minihypers in a finite projective geometry and its applications to error-correcting codes, Bull. Osaka Women's Univ. 24 (1987), 1-24. 
[23] N. Hamada, T. Helleseth, A characterization of some q-ary codes $(q>(h-$ $\left.1)^{2}, h \geq 3\right)$ meeting the Griesmer bound, Math. Japon. 38 (1993), no. 5, 925939.

[24] M. Lavrauw, L. Storme, G. Van de Voorde, On the code generated by the incidence matrix of points and $k$-spaces in $P G(n, q)$ and its dual, Finite Fields Appl. 14 (2008), no. 4, 1020-1038.

[25] G. Lunardon, Linear k-blocking sets, Combinatorica 21 (2001), 571-581.

[26] K. Metsch, L. Storme, Partial t-spreads in PG(2t+1,q), Des. Codes Cryptogr. 18 (1999), no. 1-3, 199-216.

[27] P. Polito, O. Polverino, On small blocking sets, Combinatorica 18 (1998), 133137.

[28] O. Polverino, Small minimal blocking sets and complete $k$-arcs in $P G\left(2, p^{3}\right)$, Discrete Math. 208/9 (1999), 469-476.

[29] L. Rédei, Lückenhafte Polynome über endlichen Körpern, Akadémiai Kiadó, Budapest, and Birkhäuser Verlag, Basel, 1970 (English translation: Lacunary Polynomials over Finite Fields, Akadémiai Kiadó, Budapest, and North Holland, Amsterdam, 1973).

[30] M. Svéd, Baer subspaces in the n-dimensional projective space, Lecture Notes in Math. (1983), no. 1036, 375-391.

[31] P. Sziklai, On small blocking sets and their linearity, J. Combin. Theory Ser A 115 (2008), no. 7, 1167-1182.

[32] T. Szőnyi, Blocking Sets in Desarguesian Affine and Projective Planes, Finite Fields Appl. 3 (1997), no. 3, 187-202.

[33] T. Szőnyi, Zs. Weiner, Small blocking sets in higher dimensions, J. Combin. Theory Ser. A 95 (2001), no. 1, 88-101.

[34] Zs. Weiner, Small point sets of $P G(n, q)$ intersecting each $k$-space in 1 modulo $\sqrt{q}$ points, Innov. Incidence Geom. 1 (2005), 171-180.

University of Zagreb, Faculty of Electrical Engineering and ComPuting, Unska 3, 10000 Zagreb, Croatia

E-mail address: anamari.nakic@fer.hr

Ghent University, Department of Mathematics, Krijgslaan 281, 9000 Ghent, BELGIUM

E-mail address: 1s@cage.ugent.be 